\title{
Double scaling limit for matrix models with non analytic potentials
}

\author{
M. Shcherbina \\ Institute for Low Temperature Physics, Kharkov, Ukraine
}

\begin{abstract}
We study the double scaling limit for unitary invariant ensembles of random matrices with non analytic potentials and find the asymptotic expansion for the entries of the corresponding Jacobi matrix. Our approach is based on the perturbation expansion for the string equations. The first order perturbation terms of the Jacobi matrix coefficients are expressed through the Hastings-McLeod solution of the Painleve II equation. The limiting reproducing kernel is expressed in terms of solutions of the Dirac system of differential equations with a potential defined by the first order terms of the expansion.
\end{abstract}

\section{Introduction}

Unitary invariant ensembles of random matrices or matrix models play a very important role in the random matrix theory (RMT) mainly because of its numerous links with another fields of mathematics and theoretical physics. An important advantage of these ensembles is that their special structure allows to study their limiting eigenvalue distribution with much more details, than other models of RMT.

The matrix model is defined as a set of all $n \times n$ Hermitian matrices $M$ with a probability distribution

$$
P_{n}(M) d M=Z_{n}^{-1} \exp \{-n \operatorname{Tr} V(M)\} d M
$$

where $Z_{n}$ is a normalizing constant, $V: \mathbb{R} \rightarrow \mathbb{R}_{+}$is a Hölder function satisfying the condition

$$
V(\lambda) \geq(2+\epsilon) \log (1+|\lambda|)
$$

One of important objects of the investigation in the global regime is the Normalized Counting Measure (NCM) of eigenvalues $\left\{\lambda_{j}^{(n)}\right\}_{j=1}^{n}$ of the matrix $M$. According to [5, 13] the NCM tends weakly in probability, as $n \rightarrow \infty$, to the non random limiting measure $\mathcal{N}$ known as the Integrated Density of States (IDS). The IDS is normalized to unity and it is absolutely continuous if $V^{\prime}$ satisfies the Lipshitz condition. The non-negative density $\rho(\lambda)$ is called the Density of States (DOS) of the ensemble. The IDS can be found as a unique solution of a certain variational problem [5, 13] which imply, in particularly, that if $V^{\prime}(\lambda)$ satisfies the Lipshitz conditions on the support $\sigma$ of limiting IDS, then $\rho(\lambda)$ is a solution of the following integral equation

$$
V^{\prime}(\lambda)=2 \int_{\sigma} \frac{\rho(\mu) d \mu}{\lambda-\mu}, \quad \sigma=\operatorname{supp} \mathcal{N} .
$$


While the global regime depends strongly on the form of $V$, the local eigenvalue statistics is expected to be universal. Denote by $p_{n}\left(\lambda_{1}, \ldots, \lambda_{n}\right)$ the joint eigenvalue probability density. It is known (see [15]) that

$$
p_{n}\left(\lambda_{1}, \ldots \lambda_{n}\right)=Z_{n}^{-1} \prod_{1 \leq j<k \leq n}\left(\lambda_{j}-\lambda_{k}\right)^{2} \prod_{j=1}^{n} e^{-n V\left(\lambda_{j}\right)}
$$

where $Z_{n}$ is the respective normalization factor. Let

$$
p_{l}^{(n)}\left(\lambda_{1}, \ldots, \lambda_{l}\right)=\int p_{n}\left(\lambda_{1}, \ldots, \lambda_{l}, \lambda_{l+1}, \ldots \lambda_{n}\right) d \lambda_{l+1} \ldots d \lambda_{n}
$$

be the $l$ th marginal distribution density of (1.4). Universality of local eigenvalue statistics means that if we consider some $\lambda_{0} \in \sigma$, then all marginal distribution densities after a proper rescaling (which depends on the behavior of the limiting DOS $\rho(\lambda)$ near the point $\lambda=\lambda_{0}$ ) tend to some universal limits.

The most known quantity probing universality is the large- $n$ form of the hole probability

$$
E_{n}\left(\Delta_{n}\right)=\mathbf{P}_{n}\left\{\lambda_{l}^{(n)} \notin \Delta_{n}, l=1, \ldots, n\right\},
$$

where $\mathbf{P}_{n}\{\ldots\}$ is the probability defined by the distribution (1.1), and $\Delta_{n}$ is an interval of the spectral axis, whose order of magnitude is fixed by the condition $n \mathcal{N}\left(\Delta_{n}\right) \sim 1$. For the matrix models $E_{n}\left(\Delta_{n}\right)$ can be obtained as the Fredholm determinant of a certain integral operator. This structure of the hole probability is a consequence of the structure of marginal densities and the latter can be explained by the link of matrix models with orthogonal polynomials $p_{l}^{(n)}(\lambda),(l=1, \ldots)$ on $\mathbb{R}$ associated with the weight $e^{-n V(\lambda)}$. The link is provided by the formula [15]

$$
p_{l}^{(n)}\left(\lambda_{1}, \ldots, \lambda_{l}\right)=\frac{(n-l) !}{n !} \operatorname{det}\left\|K_{n}\left(\lambda_{j}, \lambda_{k}\right)\right\|_{j, k=1}^{l},
$$

where

$$
K_{n}(\lambda, \mu)=\sum_{l=1}^{n} \psi_{l}^{(n)}(\lambda) \psi_{l}^{(n)}(\mu)
$$

is known as a reproducing kernel of an orthonormalized system

$$
\psi_{l}^{(n)}(\lambda)=e^{-n V(\lambda) / 2} P_{l-1}^{(n)}(\lambda), \quad l \in \mathbb{N},
$$

in which $P_{l}^{(n)}(\lambda)$ is a polynomial of $l$-th degree with a positive coefficient in front of $\lambda^{l}$. This polynomial is uniquely defined by the orthogonality conditions

$$
\int P_{l}^{(n)}(\lambda) P_{m}^{(n)}(\lambda) e^{-n V(\lambda)} d \lambda=\delta_{l, m}
$$

Formula (1.7) gives us

$$
\left(n^{1-\gamma} / c_{0}\right)^{\ell} p_{\ell, n}\left(\lambda_{0}+t_{1} / c_{0} n^{\gamma}, \ldots, \lambda_{0}+t_{\ell} / c_{0} n^{\gamma}\right)=\operatorname{det}\left|\mathcal{K}_{n}\left(t_{i}, t_{j}\right)\right|_{i, j=1}^{\ell},
$$

where

$$
\mathcal{K}_{n}\left(t_{1}, t_{2}\right)=n^{1-\gamma} c_{0}^{-1} K_{n}\left(\lambda_{0}+t_{1}\left(c_{0} n\right)^{-\gamma}, \lambda_{0}+t_{2}\left(c_{0} n\right)^{-\gamma}\right) .
$$

Hence we can reduce the question on the behaviour of the rescaled $\ell$ th marginal density to the question of the existence of the limit of $\mathcal{K}_{n}(s, t)$ for proper chosen $\gamma$ and $c_{0}$. 
In the bulk case $\left(\rho\left(\lambda_{0}\right) \neq 0\right)$ we choose $\gamma=1$. Then the limiting hole probability is the Fredholm determinant of the integral operator, defined by the kernel $\sin \pi\left(t_{1}-t_{2}\right) / \pi\left(t_{1}-t_{2}\right)$ on the interval $(0, s)$. This fact for the GUE was established by M. Gaudin in the early 60s [15]. The same fact was proved recently in [17, 8, for certain classes of matrix models. In terms of the reproducing kernel (1.8) this result can be formulated as

$$
\lim _{n \rightarrow \infty} \rho^{-1}\left(\lambda_{0}\right) K_{n}\left(\lambda_{0}+t_{1} / \rho\left(\lambda_{0}\right) n, \lambda_{0}+t_{2} / \rho\left(\lambda_{0}\right) n\right)=\frac{\sin \pi\left(t_{1}-t_{2}\right)}{\pi\left(t_{1}-t_{2}\right)} .
$$

The edge case of local eigenvalue statistics was studied much later even for the GUE [11, 20]. It was found that if we choose $\gamma=2 / 3$, then for the edge points $\lambda_{0}= \pm a(\sigma=[-a, a])$ the hole probability (1.6) of the GUE in the limit $n \rightarrow \infty$ is the Fredholm determinant of the integral operator, defined on the interval $(0, s)$ by the kernel

$$
\mathcal{K}\left(t_{1}, t_{2}\right)=\frac{A i\left(t_{1}\right) A i^{\prime}\left(t_{2}\right)-A i^{\prime}\left(t_{1}\right) A i\left(t_{2}\right)}{t_{1}-t_{2}},
$$

where $A i(s)$ is a standard Airy function [1]. This fact for real analytic potentials in (1.1) was obtained in [9]. In the paper [18] a more simple proof of the edge universality for the same class of potentials was given. An important advantage of the method of [18] is that it can be generalized to a class of non analytic potentials.

The case of the critical point universality with $\gamma=1 / 3$ was studied first for $V(\lambda)=\frac{1}{4} \lambda^{4}-\lambda^{2}$ by using the Riemann-Hilbert approach in [4. The same method was generalized on a class of real analytic potentials in [7] under additional assumptions that the limiting spectrum $\sigma$ consists of one interval and the density $\rho(\lambda)$ behaves like a square root near the edge points and has only one critical point inside $\sigma$ (cf. condition C3 below). But the asymptotic behaviour of the Jacobi matrix coefficients was not studied.

In the present paper we find the asymptotic behaviour of the Jacobi matrix coefficients and on the basis of this result prove universality near the critical point. We need not to assume that $V(\lambda)$ is a real analytic function. Our approach is based on the mathematical version of physical ideas proposed in [6].

Let us state our main conditions.

C1. The support $\sigma$ of the IDS of the ensemble consists of a single interval:

$$
\sigma=[-2,2]
$$

C2. $V(\lambda)$ is an even real locally Lipshitz function in $\mathbb{R}$ and there exists $\varepsilon>0$ such that $V^{(6)}(\lambda) \in L_{2}\left[\sigma_{\epsilon}\right]$, where $\sigma_{\varepsilon}=[-2-\varepsilon, 2+\varepsilon]$.

C3. The DOS $\rho(\lambda)$ has the form

$$
\rho(\lambda)=\frac{1}{2 \pi} \lambda^{2} P_{0}(\lambda) \sqrt{4-\lambda^{2}}, \quad \lambda \in[-2,2],
$$

where $P_{0}(\lambda)>0$ for $\lambda \in[-2,2]$.

C4. The function

$$
u(\lambda)=2 \int \log |\mu-\lambda| \rho(\mu) d \mu-V(\lambda)
$$

achieves its maximum if and only if $\lambda \in[-2,2]$. 
Remark 1. In fact Theorems 1 and 2 below can be proved under the condition $V^{(5)}(\lambda) \in L_{2}\left[\sigma_{\epsilon}\right]$, but the proof is more complicated. Since $V^{(4)}(0)$ is used in the limiting formulas for Theorem 1 , it is natural to expect that existence of continuous $V^{(4)}(\lambda)$ in some neighborhood of $\lambda=0$ is a necessary condition for Theorem 1. Thus condition $\mathbf{C 2}$ does not look too restrictive.

Remark 2. It is well known DOS $\rho$ of the ensemble (1.1) for $\sigma=[-2,2]$ has the form

$$
\rho(\lambda)=\frac{1}{2 \pi} \chi_{\sigma}(\lambda) P(\lambda) \sqrt{4-\lambda^{2}},
$$

where $\chi_{\sigma}(\lambda)$ is the indicator of $\sigma$ and it follows from (1.3) that $P(\lambda)$ can be represented in the form

$$
P(\lambda)=\frac{1}{\pi} \int_{\sigma} \frac{V^{\prime}(\lambda)-V^{\prime}(\mu)}{(\lambda-\mu) \sqrt{4-\mu^{2}}} d \mu,
$$

So condition C3 means that $\rho(\lambda)$, behaves like square root near the edge points and has the second order zero at $\lambda=0$.

Define a semi infinite Jacobi matrix $\mathcal{J}^{(n)}$, whose entries $J_{l+1, l}^{(n)}=J_{l, l+1}^{(n)}=J_{l}^{(n)}$ are defined by the recurrent relations

$$
J_{l}^{(n)} \psi_{l+1}^{(n)}(\lambda)+J_{l-1}^{(n)} \psi_{l-1}^{(n)}(\lambda)=\lambda \psi_{l}^{(n)}(\lambda), \quad l \in \mathbb{N}, \quad J_{0}^{(n)}=0,
$$

where $\psi_{l}^{(n)}$ is defined by (1.9). The main result of the paper is

Theorem 1. Let conditions $C 1-C 4$ be fulfilled. Then for any $k:|k| \leq n^{1 / 3} \log ^{2} n$

$$
J_{n+k}^{(n)}=1+\tilde{s}(-1)^{k} n^{-1 / 3} q\left(\frac{k}{n^{1 / 3}}\right)+\frac{k}{8 n P_{0}(2)}+r_{k},
$$

where $q(x)$ is the Hastings-McLeod solution of the Painleve II equation

$$
q^{\prime \prime}(x)=\frac{1}{2 P_{0}(0)} x q(x)+2 q^{3}(x),
$$

which is uniquely defined (see [12]) by the asymptotic conditions

$$
\lim _{x \rightarrow+\infty} q(x)=0, \quad \lim _{x \rightarrow-\infty} \frac{q(x)}{(-x)^{1 / 2}}=\frac{1}{2 P_{0}^{1 / 2}(0)},
$$

$P_{0}(\lambda)$ is defined by (1.11), $\tilde{s}=\operatorname{sign}\left(1-J_{n}^{(n)}\right)$ and remainder terms $r_{k}$ satisfy the bounds

$$
\left|r_{k}\right| \leq C\left(|k / n|^{2}+n^{-4 / 3}\right)
$$

where $C$ is some absolute constant.

To prove universality of local eigenvalue statistics we study

$$
\mathcal{K}_{n}\left(t_{1}, t_{2}\right)=n^{-1 / 3} K_{n}\left(t_{1} n^{-1 / 3}, t_{2} n^{-1 / 3}\right) .
$$


Theorem 2. Under conditions $C 1-C 4$ for any $l \in \mathbb{N}$ there exists a weak limit of the marginal density (1.5)

$$
\lim _{n \rightarrow \infty}\left(2 n^{2 / 3}\right)^{l} p_{l}^{(n)}\left(2 t_{1} / n^{2 / 3}, \ldots, 2 t_{l} / n^{2 / 3}\right)=\operatorname{det}\left\{\mathcal{K}\left(t_{i}, t_{j}\right)\right\}_{i, j=1}^{l}
$$

where

$$
\mathcal{K}\left(t_{1}, t_{2}\right)=\frac{\Psi_{1}\left(0 ; t_{1}\right) \Psi_{0}\left(0 ; t_{2}\right)-\Psi_{0}\left(0 ; t_{1}\right) \Psi_{1}\left(0 ; t_{2}\right)}{\pi\left(t_{1}-t_{2}\right)},
$$

and $\boldsymbol{\Psi}(x, t)=\left(\Psi_{0}(x ; t), \Psi_{1}(x ; t)\right)$ is a solution of the Dirac system of equations

$$
\mathcal{A} \boldsymbol{\Psi}(x, t)=t \boldsymbol{\Psi}(x, t), \quad \mathcal{A}=\left(\begin{array}{cc}
0 & 1 \\
-1 & 0
\end{array}\right) \frac{d}{d x}+\left(\begin{array}{cc}
0 & q(x) \\
q(x) & 0
\end{array}\right)
$$

with $q(x)$ defined by (1.17)-(1.18), and $\mathbf{\Psi}(x, t)$ are chosen to satisfy the asymptotic conditions

$$
\lim _{x \rightarrow-\infty}|\mathbf{\Psi}(x ; t)|=0, \quad \lim _{x \rightarrow \infty}|\boldsymbol{\Psi}(x ; t)|=1 .
$$

Corollary 1. Under conditions C1-C4 the hole probability (1.6) for $\Delta_{n}=\left[n^{-1 / 3} a, n^{-1 / 3} b\right]$ converges, as $n \rightarrow \infty$, to the Fredholm determinant of the integral operator defined in $[a, b]$ by the kernel (1.21):

$$
\lim _{n \rightarrow \infty} E_{n}\left(\left[a n^{-1 / 3}, b n^{-1 / 3}\right]\right)=\operatorname{det}(I-\mathcal{K}([a, b])) .
$$

The paper is organized as follows. In Section 2 we prove Theorems 1 and 2 . The proofs of the most of auxiliary results are given in Section 3. Some auxiliary results which have no direct links with matrix models (some properties of the Hastings-McLeod solution, bounds for smooth functions of Jacobi matrices etc.), are proven in Appendix.

\section{Proofs of Theorems 1, 2,}

Proof of Theorem 1. The main idea of the proof is to use the perturbation expansion of the string equations:

$$
J_{k}^{(n)} V^{\prime}\left(\mathcal{J}^{(n)}\right)_{k, k+1}=\frac{k}{n}
$$

which we consider as a system of nonlinear equations with respect to the coefficients $J_{k}^{(n)}$. Here and below we denote by $\mathcal{J}^{(n)}$ a semi-infinite Jacobi matrix, defined in (1.15). Relations (2.1) can be easily obtained from the identity

$$
\int\left(e^{-n V(\lambda)} P_{k+1}^{(n)}(\lambda) P_{k}^{(n)}(\lambda)\right)^{\prime} d \lambda=0 .
$$

Our first step is the following lemma, proven in Section 3 ,

Lemma 1. Under conditions $\mathbf{C 1}$ - C3 uniformly in $k:|k-n| \leq n^{1 / 2}$

$$
\left|J_{k}^{(n)}-1\right| \leq C n^{-1 / 8} \log ^{1 / 4} n, \quad\left|J_{k}^{(n)}+J_{k+1}^{(n)}-2\right| \leq C n^{-1 / 4} \log ^{1 / 2} n .
$$

Remark 3. The convergence $J_{k}^{(n)} \rightarrow 1$, as $n \rightarrow \infty$ and $|k-n|=o(n)$ without uniform bounds for the remainders was proven in [2] under much more weak conditions $\left(V^{\prime}(\lambda)\right.$ is a Hölder function in some neighborhood of the limiting spectrum). 
Denote $\mathcal{J}^{(0)}$ an infinite Jacobi matrix with constant coefficients

$$
\mathcal{J}_{k, k+1}^{(0)}=\mathcal{J}_{k+1, k}^{(0)}=1
$$

and for any positive $N<n$ define an infinite Jacobi matrix $\tilde{\mathcal{J}}(N)$ with the entries

$$
\tilde{J}_{k}= \begin{cases}J_{n+k}^{(n)}-1, & |k|<N \\ 0, & \text { otherwise. }\end{cases}
$$

Proposition 1. For any function $v(\lambda)$, whose $\ell$ th derivative belongs to $L_{2}\left[\sigma_{\varepsilon}\right]\left(\sigma_{\varepsilon}=[-2-\varepsilon, 2+\right.$ $\varepsilon]$ ), consider a periodic function $\tilde{v}(\lambda)=\tilde{v}(\lambda+4+2 \varepsilon)$ with the same number of derivatives, and such that $\tilde{v}(\lambda)=v(\lambda)$ for $|\lambda| \leq 2+\varepsilon / 2$. Let also $n^{1 / 2} \geq N, M>n^{1 / 3}$ and $\tilde{\mathcal{J}}(N+M)$ is defined by (2.3). Then uniformly in $N, M$ and $|k| \leq N$ for any fixed integer $\delta$

$$
v\left(\mathcal{J}^{(n)}\right)_{n+k, n+k+\delta}-\tilde{v}\left(\mathcal{J}^{(0)}+\tilde{\mathcal{J}}(N+M)\right)_{k, k+\delta}=O\left(M^{-\ell+1 / 2}\right) .
$$

The proof of the proposition is given in Appendix.

To estimate the remainder terms of our expansion we define

$$
m_{k}:=\max \left\{\max _{|j| \leq|k|+n^{1 / 3} / 2}\left\{\left|\tilde{J}_{j}\right|,\left|\tilde{J}_{j}+\tilde{J}_{j+1}\right|^{1 / 2}\right\},(|k| / n)^{1 / 2}\right\} .
$$

Lemma 2. Let $v(\lambda)$ satisfy conditions of Proposition 1 with $\ell=5, \delta$ be any fixed integer and $|k| \leq 3 n^{1 / 2} / 4$. Then

$$
\begin{aligned}
v\left(\mathcal{J}^{(n)}\right)_{n+k, n+k+\delta}= & v\left(\mathcal{J}^{(0)}\right)_{k, k+\delta}-c_{1}^{(\delta)} \tilde{J}_{k}+\sum^{\prime} \mathcal{P}_{k-l_{1}}^{(\delta)} \tilde{J}_{l_{1}}+\sum^{\prime} \mathcal{P}_{l_{1}, l_{2}}^{(2, k, \delta)} \tilde{J}_{l_{1}} \tilde{J}_{l_{2}} \\
& +\sum^{\prime} \mathcal{P}_{l_{1}, l_{2}, l_{3}}^{(3, \delta)} \tilde{J}_{l_{1}} \tilde{J}_{l_{2}} \tilde{J}_{l_{3}}+r_{k}^{(\delta)} \\
= & v\left(\mathcal{J}^{(0)}\right)_{k, k+\delta}-c_{1}^{(\delta)} \tilde{J}_{k}+\Sigma^{(1)}+\Sigma^{(2)}+\Sigma^{(3)}+r_{k}^{(\delta)}
\end{aligned}
$$

where $\left|r_{k}^{(\delta)}\right| \leq C m_{k}^{4}$ and $\mathcal{P}_{l_{1}, l_{2}}^{(2, k, \delta)}$ and $\mathcal{P}_{l_{1}, l_{2}, l_{3}}^{(3, k, k}$ satisfy the bounds:

$$
\mid \begin{aligned}
& \sum^{\prime} \mathcal{P}_{l_{1}, l_{2}}^{(2, k, \delta)}\left(l_{1}-k\right)\left(l_{2}-k\right) \tilde{x}_{l_{1}} \tilde{y}_{l_{2}} \mid \leq C\|\tilde{x}\|\|\tilde{y}\|, \\
& \sum^{\prime} \mathcal{P}_{l_{1}, l_{2}}^{(2, k, \delta)}\left(l_{1}-k\right)^{2} \tilde{x}_{l_{1}} \tilde{y}_{l_{2}} \mid \leq C\|\tilde{x}\|\|\tilde{y}\|, \\
& \sum^{\prime} \mathcal{P}_{l_{1}, l_{2}, l_{3}}^{(3, k, \delta)}\left(l_{1}-k\right) \tilde{x}_{l_{1}} \tilde{y}_{l_{2}} \tilde{z}_{l_{3}} \mid \leq C\|\tilde{x}\|\|\tilde{y}\|\|\tilde{z}\|
\end{aligned}
$$

for any bounded sequences $\left\{\tilde{x}_{k}\right\},\left\{\tilde{y}_{k}\right\}$ and $\left\{\tilde{z}_{k}\right\}$. Here and below $\|x\|=\max _{k}\left|x_{k}\right|$ and $\sum^{\prime} \operatorname{means}$ the summation over indexes $\left|l_{i}\right| \leq|k|+n^{1 / 3} / 2$

Moreover,

$$
\mathcal{P}_{l}^{(\delta)}=\frac{1}{2 \pi} \int_{-\pi}^{\pi} F^{(\delta)}(2 \cos (x / 2)) e^{i l x} d x
$$

with some smooth $F^{(\delta)}(\lambda)$ and for $\delta=1$

$$
c_{1}^{(1)}=\frac{1}{2 \pi} \int_{-\pi}^{\pi} v(2 \cos x) \cos x d x, \quad F^{(1)}(\lambda)=2 P(\lambda)=\frac{2}{\pi} \int_{-2}^{2} \frac{v(\lambda)-v(\mu)}{(\lambda-\mu) \sqrt{4-\mu^{2}}} d \mu .
$$

For $\delta=0$

$$
c_{1}^{(0)}=0, \quad F^{(0)}(2 \cos (x / 2))=\frac{\cos ^{2}(x / 2)}{2 \pi} \int_{-\pi}^{\pi} \frac{v\left(2 \cos x_{1}\right) d x_{1}}{\cos ^{2} x_{1}-\cos ^{2}(x / 2)} .
$$


The proof of the lemma is given in Section 3 .

Remark 4. If $v$ coinsides with $V^{\prime}$ for $\lambda \in \sigma_{\varepsilon / 2}$, then

$$
c_{1}=\frac{1}{2 \pi} \int_{-\pi}^{\pi} v(2 \cos x) \cos x d x=\frac{1}{\pi} \int_{-\pi}^{\pi} d x \int_{-2}^{2} \cos x \frac{\rho(\lambda) d \lambda}{2 \cos x-\lambda}=1 .
$$

Remark 5. If in (2.9) $P(\lambda)=\lambda^{2} P_{0}(\lambda)$, then for any $\tilde{x}_{k}$

$$
\sum \mathcal{P}_{k-l}^{(1)} \tilde{x}_{l}=\sum \mathcal{P}_{k-l}^{(0)}\left(\tilde{x}_{l+1}+2 \tilde{x}_{l}+\tilde{x}_{l-1}\right)
$$

where

$$
\mathcal{P}_{l}^{(0)}=\frac{1}{\pi} \int_{-\pi}^{\pi} P_{0}(2 \cos (x / 2)) e^{i l x} d x
$$

Denote

$$
y_{k}=k /\left(8 P_{0}(2) n\right),
$$

where $P_{0}(\lambda)$ is defined by (1.11). Then we represent $\tilde{J}_{k}$ in the form

$$
\tilde{J}_{k}=(-1)^{k} x_{k}+y_{k}, \quad \text { or } \quad x_{k}=(-1)^{k}\left(\tilde{J}_{k}-y_{k}\right) .
$$

Now, substituting (2.14) in (2.6) and keeping the terms up to the order $m_{k}^{3}$ (recall, that by definition (2.5) $\left.y_{k}=O\left(m_{k}^{2}\right)\right)$, we get for $\delta=1$

$$
\begin{aligned}
\Sigma^{(1)} & =-\sum^{\prime} \mathcal{P}_{k-l}^{(0)}(-1)^{l} d_{l}^{(2)}+\sum^{\prime} \mathcal{P}_{k-l}^{(1)} y_{l}=-\Sigma_{1}^{(1)}+\Sigma_{2}^{(1)}, \\
\Sigma^{(2)} & =\sum^{\prime} \mathcal{P}_{l_{1}, l_{2}}^{(2,1)}(-1)^{l_{1}+l_{2}} x_{l_{1}} x_{l_{2}}+2 \sum^{\prime} \mathcal{P}_{l_{1}, l_{2}}^{(2, k, 1)}(-1)^{l_{1}} x_{l_{1}} y_{l_{2}}+O\left(m_{k}^{4}\right) \\
& =\Sigma_{1}^{(2)}+\Sigma_{2}^{(2)}+O\left(m_{k}^{4}\right), \\
\Sigma^{(3)} & =\sum^{\prime} \mathcal{P}_{l_{1}, l_{2}, l_{3}}^{(3, k)}(-1)^{l_{1}+l_{2}+l_{3}} x_{l_{1}} x_{l_{2}} x_{l_{3}}+O\left(m_{k}^{4}\right)=\Sigma_{1}^{(3)}+O\left(m_{k}^{4}\right) .
\end{aligned}
$$

Here and below we denote

$$
d_{k}^{(1)}=x_{k+1}-x_{k}, \quad d_{k}^{(2)}=d_{k}^{(1)}-d_{k-1}^{(1)}, \quad d_{k}^{(3)}=d_{k+1}^{(2)}-d_{k}^{(2)} .
$$

Since by definition (2.5) $\left|d_{k}^{(1)}\right| \leq C m_{k}^{2}$, using bounds (2.7), we can write

$$
\begin{aligned}
\Sigma_{1}^{(2)}= & x_{k}^{2} \sum^{\prime} \mathcal{P}_{l_{1}, l_{2}}^{(2, k, 1)}(-1)^{l_{1}+l_{2}}+2 x_{k} d_{k}^{(1)} \sum^{\prime} \mathcal{P}_{l_{1}, l_{2}}^{(2, k, 1)}(-1)^{l_{1}+l_{2}}\left(l_{1}-k\right) \\
& +2 x_{k} \sum^{\prime} \mathcal{P}_{l_{1}, l_{2}}^{(2, k, 1)}(-1)^{l_{1}+l_{2}}\left(\left(x_{l_{1}}-x_{k}\right)-\left(l_{1}-k\right) d_{k}^{(1)}\right)+O\left(m_{k}^{4}\right) \\
= & x_{k}^{2} \Sigma_{1,1}^{(2)}+2 x_{k} d_{k}^{(1)} \Sigma_{1,2}^{(2)}+2 x_{k} \Sigma_{1,3}^{(2)}+O\left(m_{k}^{4}\right) .
\end{aligned}
$$

Similarly

$$
\begin{aligned}
& \Sigma_{2}^{(2)}=x_{k} y_{k} \sum^{\prime} \mathcal{P}_{l_{1}, l_{2}}^{(2, k, 1)}(-1)^{l_{1}}+O\left(m_{k}^{4}\right)=x_{k} y_{k} \Sigma_{2,1}^{(2)}+O\left(m_{k}^{4}\right) \\
& \Sigma_{1}^{(3)}=x_{k}^{3} \sum^{\prime} \mathcal{P}_{l_{1}, l_{2}, l_{3}}^{(3, k}(-1)^{l_{1}+l_{2}+l_{3}}+O\left(m_{k}^{4}\right)=x_{k}^{3} \Sigma_{1,1}^{(3)}+O\left(m_{k}^{4}\right)
\end{aligned}
$$

and

$$
\Sigma_{1}^{(1)}=-d_{k}^{(2)} \sum^{\prime} \mathcal{P}_{k-l}^{(0)}(-1)^{l}-\sum^{\prime} \mathcal{P}_{k-l}^{(0)}(-1)^{l}\left(d_{l}^{(2)}-d_{k}^{(2)}\right)=-d_{k}^{(2)} \Sigma_{1,1}^{(1)}-\Sigma_{1,2}^{(1)}
$$


Proposition 2. If $v(\lambda)=V^{\prime}(\lambda)$, as $\lambda \in \sigma_{\varepsilon / 2}$, then

$$
\begin{array}{ll}
\Sigma_{1,1}^{(1)}=2(-1)^{k} P_{0}(0)+O\left(n^{-5 / 6}\right), & \Sigma_{2}^{(1)}=k / n+O\left(n^{-3 / 2}\right) \\
\Sigma_{1,1}^{(2)}=1+O\left(n^{-5 / 6}\right), & \Sigma_{1,2}^{(2)}=O\left(n^{-1 / 2}\right) \\
\Sigma_{2,1}^{(2)}=(-1)^{k}+O\left(n^{-5 / 6}\right), & \Sigma_{1,1}^{(3)}=(-1)^{k}\left(4 P_{0}(0)-1\right)+O\left(n^{-1 / 2}\right)
\end{array}
$$

If $v^{(0)}(\lambda)=\lambda^{-1} V^{\prime}(\lambda)$ for $\lambda \in \sigma_{\varepsilon / 2}$, then

$$
\sum^{\prime} \mathcal{P}_{l-k}^{(0)}=4 P_{0}(2)+O\left(n^{-5 / 6}\right), \quad \sum^{\prime} \mathcal{P}_{l_{1}, l_{2}}^{(2, k, 0)}(-1)^{l_{1}+l_{2}}=2 P_{0}(0)+O\left(n^{-5 / 6}\right) .
$$

Substituting (2.19) into (2.17)-(2.18), we obtain

$$
\begin{aligned}
V^{\prime}\left(\mathcal{J}^{(n)}\right)_{n+k, n+k+1}= & 1-(-1)^{k} x_{k}-y_{k}-\sum^{\prime} \mathcal{P}_{k-l}^{(0)}(-1)^{l} d_{l}^{(2)}+k / n \\
& +x_{k}^{2}+2 x_{k} \Sigma_{1,3}^{(2)}+2 x_{k} y_{k}(-1)^{k}+(-1)^{k}\left(4 P_{0}(0)-1\right) x_{k}^{3}+O\left(m_{k}^{4}\right)
\end{aligned}
$$

Using this expression in (2.1) and keeping the terms up to the order $O\left(m_{k}^{3}\right)$, we get

$$
-\sum^{\prime} \mathcal{P}_{k-l}^{(0)}(-1)^{(l-k)} d_{l}^{(2)}+4 P_{0}(0) x_{k}^{3}+8 P_{0}(2) x_{k} y_{k}+2 x_{k} \Sigma_{1,3}^{(2)}=O\left(m_{k}^{4}\right)
$$

Remark 6. If the operator $\mathcal{P}^{(0)}$ has the form (2.12) with $P_{0}(x)>d>0$, then there exists

$$
\left(\mathcal{P}^{(0)}\right)_{k, l}^{-1}=\frac{1}{4 \pi} \int_{-\pi}^{\pi} P_{0}^{-1}(2 \cos (x / 2)) e^{i(k-l) x} d x .
$$

So, if for some $z_{j}$ we have the system of equations

$$
\sum \mathcal{P}_{k-l}^{(0)} z_{l}=\varepsilon_{k}, \quad k \in \mathbb{Z},
$$

then

$$
\left|z_{k}\right| \leq \sum_{l}\left|\left(\mathcal{P}^{(0)}\right)_{k-l}^{-1}\right|\left|\varepsilon_{l}\right|
$$

We apply this remark to (2.22) written in the form (2.23) with $z_{k}=(-1)^{k} d_{k}^{(2)}$, where $d_{k}^{(2)}$ are defined by (2.16) with $x_{k}$ of the form (2.14) for $|k| \leq n^{1 / 2}$ and $x_{k}=0$ for $k>n^{1 / 2}$. We take also

$$
\varepsilon_{k}= \begin{cases}4 P_{0}(0) x_{k}^{3}+8 P_{0}(2) x_{k} y_{k}+2 x_{k} \Sigma_{1,3}^{(2)}+\sum_{|l|>N_{k}} P_{k-l}^{(0)}(-1)^{l} d_{l}^{(2)}+O\left(m_{k}^{4}\right), & k \leq 3 n^{1 / 2} / 4 \\ \sum P_{k-l}^{(0)}(-1)^{l} d_{l}^{(2)} & \text { otherwise }\end{cases}
$$

Since $P^{(0)}(\lambda)$ has the third derivative (see conditions C2, C3 and representation (1.14),$\left(P^{(0)}(\lambda)\right)^{-1}$ also does. Hence, using the standard bound for the remainder of the Fourier expansion of the functions $P^{(0)}(\lambda)$ and $\left(P^{(0)}(\lambda)\right)^{-1}$ (see, e.g. (4.2) ), we have for any $M$

$$
\sum_{|l|>M}\left|\mathcal{P}_{l}^{(0)}\right| \leq C M^{-5 / 2}, \quad \sum_{|l|>M}\left|\left(\mathcal{P}^{(0)}\right)_{l}^{-1}\right| \leq C M^{-5 / 2}
$$

Using the first of these bounds and the inequality

$$
\left|d_{k}^{(2)}\right|=\left|d_{k}^{(1)}-d_{k}^{(1)}\right| \leq \begin{cases}C n^{-1 / 4} \log ^{1 / 4} n, & || k\left|-n^{1 / 2}\right|>2 \\ C n^{-1 / 8} \log ^{1 / 8} n, & \text { otherwise }\end{cases}
$$


which follows from Lemma 1, we get

$$
\left|\varepsilon_{k}\right| \leq C \begin{cases}m_{k}^{3}+n^{-13 / 12} \log ^{1 / 2} n, & |k| \leq n^{1 / 2} / 2, \\ n^{-1 / 4} \log ^{1 / 2} n & n^{1 / 2} / 2<|k| \leq n^{1 / 2} / 2 \\ C n^{-1 / 8} \log ^{1 / 4} n, & \text { otherwise. }\end{cases}
$$

Using this bound in the l.h.s. of (2.24) and taking into account the second bound in (2.26), we obtain

$$
\left|d_{k}^{(2)}\right| \leq C m_{k+\left[n^{1 / 3} / 4\right]}^{3}, \quad k \leq n^{1 / 2} / 3 .
$$

Hence, using this bound in (2.24) and (2.7), we get that $x_{k} \Sigma_{1,3}^{(2)}$ (see (2.17)) is less than $O\left(m_{k+\left[n^{1 / 3} / 4\right]}^{4}\right)$, and therefore (2.22) can be rewritten as

$$
-\sum^{\prime} \mathcal{P}_{k-l}^{(0)}(-1)^{l} d_{l}^{(2)}+4 P_{0}(0) x_{k}^{3}+8 P_{0}(2) x_{k} y_{k}=O\left(m_{k+\left[n^{1 / 3} / 4\right]}^{4}\right) .
$$

Now subtracting from (2.29) the same equation written for $k:=k-1$, we get

$$
\sum^{\prime} \mathcal{P}_{k-l}^{(0)}(-1)^{l} d_{l}^{(3)}=O\left(m_{k+\left[n^{1 / 3} / 4\right]}^{4}\right) .
$$

Using Remark [6 by the way described above, we obtain that $\left|d_{k}^{(3)}\right| \leq C m_{k+\left[n^{1 / 3} / 2\right]}^{4}$ for $|k| \leq$ $n^{1 / 2} / 4$. Hence, writing

$$
\sum^{\prime} \mathcal{P}_{k-l}^{(0)}(-1)^{l} d_{l}^{(2)}=d_{k}^{(2)} \Sigma_{1,1}^{(1)}+\sum^{\prime} \mathcal{P}_{k-l}^{(0)}(-1)^{l}\left(d_{l}^{(2)}-d_{k}^{(2)}\right)=d_{k}^{(2)} \Sigma_{1,1}^{(1)}+O\left(m_{k+\left[n^{1 / 3} / 2\right]}^{4}\right),
$$

in view of the first relation in (2.19), we get from (2.29)

$$
d_{k}^{(2)}-2 x_{k}^{3}-\frac{k}{2 P_{0}(0) n} x_{k}=r_{k}, \quad\left|r_{k}\right| \leq \tilde{C}^{\prime} \tilde{m}_{k}^{4}, \quad \text { where } \quad \tilde{m}_{k}:=m_{k+\left[n^{1 / 3} / 2\right]} .
$$

Lemma 3. There exist $C^{*}, L^{*}>0$ such that for any $k: n^{1 / 2} / 5>|k|>L^{*} n^{1 / 3}$

$$
\tilde{m}_{k} \leq C^{*}(|k| / n)^{1 / 2} .
$$

Besides, there exist $C_{1,2,3}$ such that for $n^{1 / 3}<k<k^{*}=\left[n^{1 / 3} \log ^{2} n\right]$

$$
\left|x_{k}\right| \leq C_{1} n^{-1 / 3} e^{-C_{2}\left(k / n^{1 / 3}\right)^{3 / 2}}+C_{3} \tilde{m}_{2 k^{*}}^{4}
$$

The proof is given in the next section. It is based on the proposition proven in Appendix.

Proposition 3. Let $\left\{\tilde{x}_{k}\right\}_{|k|<M}$, satisfy the recursive relations:

$$
\tilde{x}_{k+1}-2 \tilde{x}_{k}+\tilde{x}_{k-1}=2 \tilde{x}_{k}^{3}+\tilde{r}_{k}, \quad\left|\tilde{r}_{k}\right| \leq \varepsilon^{3}, \quad\left|\tilde{x}_{k}\right| \leq \varepsilon_{1} .
$$

Then for any $|k|<M-2 M_{1}$ with $M_{1}>2 \varepsilon^{-1} / 3$

$$
\left|\tilde{x}_{k}\right| \leq \max \left\{\varepsilon,\left(2 M_{1}^{-2} \varepsilon_{1}\right)^{1 / 3}\right\}, \quad\left|\tilde{x}_{k+1}-\tilde{x}_{k}\right| \leq 4 \max \left\{\varepsilon^{2},\left(2 M_{1}^{-2} \varepsilon_{1}\right)^{2 / 3}\right\} .
$$

If for $|k| \leq M$

$$
\tilde{x}_{k+1}-2 \tilde{x}_{k}+\tilde{x}_{k-1}=f_{k} \tilde{x}_{k}+\tilde{r}_{k}
$$

with $f_{k} \geq d^{2}>0$, then for $|k|<M$

$$
\left|\tilde{x}_{k}\right| \leq C \sum_{|j| \leq M} e^{-d|k-j|} \tilde{r}_{j}+\left|x_{M}\right| e^{-d|M-k|}+\left|x_{-M}\right| e^{-d|M+k|} .
$$


Notice that Lemma 2 combined with Lemma 3 give us a useful corollary

Corollary 2. For any function $\phi(\lambda)$ which has two bounded derivatives on $[-2+\varepsilon, 2+\varepsilon]$

$$
\left|\phi\left(\mathcal{J}^{(n)}\right)_{n+k, n+k}-\frac{1}{\pi} \int_{-2}^{2} \frac{\phi(\lambda) d \lambda}{\sqrt{4-\lambda^{2}}}\right| \leq C(|k| / n)^{1 / 2}+O\left(n^{-1 / 3}\right) .
$$

Now let us define a continuous function $q_{n}(x)$, which for $x \in \mathbb{Z} / n^{1 / 3}$ coincides with $x_{k}$

$$
q_{n}\left(\frac{k}{n^{1 / 3}}\right)=n^{1 / 3} x_{k}
$$

and is a linear function for $x \notin \mathbb{Z} / n^{1 / 3}$. Lemma 3 allows us to write (2.30) as

$$
\frac{q_{n}\left(\frac{k+1}{n^{1 / 3}}\right)-2 q_{n}\left(\frac{k}{n^{1 / 3}}\right)+q_{n}\left(\frac{k-1}{n^{1 / 3}}\right)}{n^{-2 / 3}}=2 q_{n}^{3}\left(\frac{k}{n^{1 / 3}}\right)+\frac{1}{2 P_{0}(0)} \frac{k}{n^{1 / 3}} q_{n}\left(\frac{k}{n^{1 / 3}}\right)+O\left(n^{-1 / 6}\right),
$$

where the bound on the remainder is uniform in $|k| / n^{1 / 3} \leq L$ for any $L$. We are interested in the behaviour of the solution of this discrete equations which satisfies conditions (cf. (2.31) and (2.32)):

$$
\left|q_{n}(x)\right| \leq C|x|^{1 / 2}, \quad\left|q_{n}(x)\right| \leq e^{-C x^{3 / 2} / 2}, \quad \text { as } \quad x \rightarrow+\infty .
$$

It follows from Lemma 3 that the functions $\left\{q_{n}(x)\right\}_{n=1}^{\infty}$ are uniformly bounded and equicontinuous for any bounded interval. Hence, this family is weakly compact in any compact set in $\mathbb{R}$ and any convergent subsequence converges uniformly to some solution of the Painleve equation (1.17), satisfying (2.39). Now we need to prove the asymptotic relations (1.18) for $x \rightarrow-\infty$. To this aim we use Lemma 4 below, which describes the behavior of the Stieltjes transform of the following densities (cf (1.7)-(1.8) )

$$
\begin{gathered}
K_{k, n}(\lambda, \mu)=\sum_{l=1}^{k} \psi_{l}^{(n)}(\lambda) \psi_{l}^{(n)}(\mu), \\
\rho_{k, n}(\lambda)=n^{-1} K_{k, n}(\lambda, \lambda), \quad g_{k, n}(z)=\int \frac{\rho_{k, n}(\lambda) d \lambda}{z-\lambda} .
\end{gathered}
$$

Lemma 4. For any $k:|k| \leq n^{1 / 3} \log ^{2} n g_{n+k, n}(z)$ can be represented in the form

$$
\begin{aligned}
g_{n+k, n}(z)= & \frac{1}{2}\left(V^{\prime \prime}(0) z+\frac{V^{(4)}(0)}{6} z^{3}\right) \\
& -\frac{1}{2} X(z)\left(P_{0}^{2}(0) z^{4}+\frac{k}{n} P_{0}(0) z^{2}+c_{k}-\delta_{n+k, n}(z)-\tilde{\delta}_{n+k, n}(z)\right)^{1 / 2},
\end{aligned}
$$

where $X(z)=\sqrt{z^{2}-4}$ (here and below we choose the branch which behaves like $z$ as $z \rightarrow+\infty$ ) and

$$
c_{k}= \pm n^{-5 / 3} \sum_{j=0}^{|k|}\left(2 P_{0}(0) q_{n}^{2}\left(\frac{ \pm j}{n^{1 / 3}}\right) \pm \frac{j}{2 n^{1 / 3}}\right)+n^{-5 / 6} O\left((|k| / n)^{3 / 2}\right) .
$$

( \pm corresponds to the sign of $k$ ). Moreover, the remainder terms $\delta_{n+k, n}(z)$ and $\tilde{\delta}_{n+k, n}(z)$ in (2.41) for $z:|z|<1$ admit the bounds

$$
\begin{aligned}
\left|\delta_{n+k, n}(z)\right| & =\left|n^{-2} \int \frac{K_{n+k, n}^{2}\left(\lambda_{1}, \lambda_{2}\right)\left(\lambda_{1}-\lambda_{2}\right)^{2}}{\left(\lambda_{1}-z\right)^{2}\left(\lambda_{2}-z\right)^{2}} d \lambda_{1} d \lambda_{2}\right| \\
& \leq \frac{C}{n^{2}|\Im z|^{2}}\left(1+\frac{|k|}{n|\Im z|^{2}}+\frac{1}{n|\Im z|^{3}}\right)^{2}, \\
\left|\tilde{\delta}_{n+k, n}(z)\right| & \leq C\left[|z|^{2}\left(\frac{k^{2}}{n^{2}}+n^{-2 / 3}\right)+n^{-4 / 3}+\frac{\log ^{1 / 2} n}{n^{1 / 2}} \frac{|z|^{4}}{|\Im z|}+|z|^{5}\right] .
\end{aligned}
$$


The proof of the lemma is given in the next section.

Let us take $k=-\left[L n^{1 / 3}\right]$ with $L$ big enough. Since it is known (see [12]) that any solution of the Painleve II equations which satisfies (2.39) assumes also the bound

$$
q^{2}(x) \leq \frac{-x}{4 P_{0}(0)}, \quad x \leq-L_{0}
$$

we can conclude that

$$
0 \leq c_{k} \leq \frac{k^{2}}{4 n^{2}}+O\left((|k| / n)^{5 / 2}\right) .
$$

Now let us choose $\tilde{\varepsilon}=n^{-1 / 3} P_{0}^{-1 / 2}(0)$ and put in (2.41) $z=\tilde{\varepsilon} \zeta$. Then (2.41) takes the form

$$
g_{n+k, n}(\tilde{\varepsilon} \zeta)=\tilde{V}(\zeta)-\frac{1}{2} \tilde{\varepsilon}^{2} P_{0}(0) X(\tilde{\varepsilon} \zeta) \sqrt{\zeta^{4}-\zeta^{2}+\tilde{c}_{k}+\tilde{\phi}(\zeta)}
$$

where $\tilde{V}$ is an analytic function,

$$
0 \leq \tilde{c}_{k}=P_{0}^{-2}(0) \tilde{\varepsilon}^{-4} c_{k} \leq \frac{L^{2}}{4},
$$

(see (2.44) $)$, and

$$
|\tilde{\phi}(\zeta)|=P_{0}^{-2}(0) \tilde{\varepsilon}^{-4}\left|\delta_{k, n}(\tilde{\varepsilon} \zeta)+\tilde{\delta}_{k, n}(\tilde{\varepsilon} \zeta)+O\left(k n^{-2}\right)\right| \leq C\left(1+|\zeta|^{2}\right),
$$

for $|\Im \zeta| \geq 1$ (see (2.43) $)$. Let $b$ be the smallest root of the quadratic equation

$$
\zeta^{2}-L \zeta+\tilde{c}_{k}=0
$$

We note, that due to (2.46) $b$ is real and positive. Consider

$$
\begin{aligned}
I(b, L)=\frac{\tilde{\varepsilon}^{-2}}{2 \pi i} \oint_{\mathcal{L}} g_{n+k, n}(\tilde{\varepsilon} \zeta) e^{-\zeta^{2} / 2} d \zeta & \\
& =\frac{P_{0}(0)}{2 \pi i} \oint_{\mathcal{L}} X(\tilde{\varepsilon} \zeta) \sqrt{\left(\zeta^{2}-b\right)\left(\zeta^{2}-L+b\right)} e^{-\zeta^{2} / 2} d \zeta+\tilde{r}_{L},
\end{aligned}
$$

where $\mathcal{L}$ consists of two lines $\Im \zeta= \pm 1$ and

$$
\left|\tilde{r}_{L}\right| \leq C \oint_{\mathcal{L}} \frac{|\phi(\zeta)| \cdot|X(\tilde{\varepsilon} \zeta)| e^{-|\zeta|^{2} / 2}|d \zeta|}{\sqrt{\left(\zeta^{2}-b\right)\left(\zeta^{2}-L+b\right)}} \leq C L^{-1 / 2} .
$$

Then, using the Cauchy theorem, we get

$$
\begin{aligned}
I(b, L)= & \frac{P_{0}(0)}{2 \pi} \Im \int \sqrt{\left((\varepsilon x)^{2}-4\right)\left(x^{2}-b\right)\left(x^{2}-L+b\right)} e^{-x^{2} / 2} d x+\tilde{r}_{L} \\
= & \frac{P_{0}(0)}{2 \pi}\left(-\int_{|x|<b}+\int_{|x| \geq L-b}\right) \sqrt{\left(x^{2}-b\right)\left(x^{2}-L+b\right)} e^{-x^{2} / 2} d x+\tilde{r}_{L}+O(\tilde{\varepsilon}) \\
& =P_{0}(0) I_{1}(b, L)+\tilde{r}_{L}+O(\tilde{\varepsilon}) .
\end{aligned}
$$

One can prove easily that for large $L$

$$
I_{1}(b, L) \sim-C_{0} L^{1 / 2} b^{3 / 2}, \quad\left(C_{0}>0\right) .
$$


On the other hand,

$$
I(b, L)=-\frac{\tilde{\varepsilon}^{-2}}{2 \pi} \int e^{-x^{2} / 2 \sigma} \lim _{\varepsilon \rightarrow 0} \Im g_{n+k, n}(\tilde{\varepsilon} \zeta) d x>0 .
$$

Thus, taking into account (2.48)

$$
L^{1 / 2} b^{3 / 2} \leq C^{\prime}\left|\tilde{r}_{L}\right| \leq C^{\prime \prime} L^{-1 / 2} \Rightarrow\left|c_{k}\right|=P_{1}^{2}(0) \tilde{\varepsilon}^{4}(L-b) b / 4 \leq C_{1}^{\prime} n^{-4 / 3} L^{1 / 3} .
$$

The last inequality combined with (2.42), and the bound for the first differences $d_{j}^{(1)}$ imply for $k=\left[L n^{1 / 3}\right], l=\left[L^{-1 / 6} n^{1 / 3}\right]$

$$
\begin{aligned}
n^{-4 / 3} O\left(L^{1 / 3}\right)=c_{-k}-c_{-k-l}=\frac{2 P_{0}(0)}{n^{5 / 3}} & \sum_{j=k}^{k+l} q_{n}^{2}\left(-\frac{j}{n^{1 / 3}}\right)-\frac{L+L^{-1 / 6}}{2 n^{4 / 3}} L^{-1 / 6} \\
& =n^{-4 / 3}\left[L^{-1 / 6}\left(\left(2 P_{0}(0) q_{n}^{2}\left(-\frac{k}{n^{1 / 3}}\right)-\frac{L}{2}+O\left(L^{-1 / 6}\right)\right)\right] .\right.
\end{aligned}
$$

Therefore,

$$
\left|q_{n}(-L)\right|=\left(4 P_{0}(0)\right)^{-1 / 2} L^{1 / 2}\left(1+O\left(L^{-1 / 2}\right)\right) .
$$

But it is easy to show that any bounded for positive $x$ solution of (1.17), which possesses the above property satisfies also the asymptotic relations

$$
q_{n}(-L)=\tilde{s}\left(4 P_{0}(0)\right)^{-1 / 2} L^{1 / 2}\left(1+O\left(L^{-2}\right)\right), \quad \tilde{s}=\operatorname{sign} q(0) .
$$

Hence, we have proved (1.18) and now can conclude that $q_{n}(x)$ converge uniformly on any compact in $\mathbb{R}$ to the Hastings-McLeod solution of (1.17), so that

$$
\Delta_{n}(x)=q_{n}(x)-q(x) \rightarrow 0 \text {, as } n \rightarrow \infty .
$$

But from (2.38) we derive that for any $x=k / n^{1 / 3}$ and $h=n^{-1 / 3}$ we have $h^{-2}\left(\Delta_{n}(x+h)+\Delta_{n}(x-h)-2 \Delta_{n}(x)\right)=\left[2 q_{n}^{2}(x)+2 q^{2}(x)+2 q_{n}(x) q(x)+\frac{x}{2 P_{0}(0)}\right] \Delta_{n}(x)+r(x)$.

and uniformly in $n$

$$
\left|\Delta_{n}(x)\right| \rightarrow 0 \text {, as } x \rightarrow \pm \infty \text {. }
$$

Proposition 4. For the Hastings - McLeod solution of (1.17) there exists $\delta>0$ such that

$$
6 q^{2}(x)+\frac{x}{2 P_{0}(0)} \geq \delta^{2}
$$

This proposition allows us to apply the assertion (2.36) of Proposition 3 to $\tilde{x}_{k}=\Delta\left(k / n^{1 / 3}\right)$ with $d=n^{-1 / 3} \delta$ and $\tilde{r}_{k}=r_{k}-r_{k}^{\prime}$ with $r_{k}$ from (2.30) and

$$
r_{k}^{\prime}=q\left(\frac{k+1}{n^{1 / 3}}\right)-2 q\left(\frac{k}{n^{1 / 3}}\right)+q\left(\frac{k-1}{n^{1 / 3}}\right)-2 n^{-2 / 3} q^{3}\left(\frac{k}{n^{1 / 3}}\right)+\frac{4}{2 n P_{0}(0)} q\left(\frac{k}{n^{1 / 3}}\right)=O\left(n^{-1}\right)
$$

uniformly in $k$. The assertion of Theorem 1 follows. 
Proof of Theorem 2. Take some fixed $\zeta_{1}, \zeta_{2}$ with $\zeta_{1,2} \neq 0$, denote $z_{1,2}=\zeta_{1,2} n^{-1 / 3}$ and consider the functions:

$$
\begin{aligned}
F_{n}\left(\zeta_{1}, \zeta_{2}\right) & =\int\left(\lambda_{1}-z_{1}\right)^{-1}\left(\lambda_{2}-z_{2}\right)^{-1}\left(\lambda_{1}-\lambda_{2}\right)^{2} K_{n}^{2}\left(\lambda_{1}, \lambda_{2}\right) d \lambda_{1} d \lambda_{2} \\
F_{n}^{(1)}\left(\zeta_{1}\right) & =n^{-2 / 3} \int\left(\lambda_{1}-z_{1}\right)^{-2}\left(\lambda_{2}-z_{1}\right)^{-2}\left(\lambda_{1}-\lambda_{2}\right)^{2} K_{n}^{2}\left(\lambda_{1}, \lambda_{2}\right) d \lambda_{1} d \lambda_{2}
\end{aligned}
$$

Changing variables $\lambda_{1,2}=t_{1,2} n^{-1 / 3}$, and using (1.20), we get

$$
F_{n}\left(\zeta_{1}, \zeta_{2}\right)=\int\left(t_{1}-\zeta_{1}\right)^{-1}\left(t_{2}-\zeta_{2}\right)^{-1}\left(t_{1}-t_{2}\right)^{2} \mathcal{K}_{n}^{2}\left(t_{1}, t_{2}\right) d t_{1} d t_{2}
$$

The proof of Theorem 2 is based on the following proposition:

Proposition 5. Let the functions $F_{n}$ and $F_{n}^{(1)}$ be defined by (2.53) and there exists $F\left(\zeta_{1}, \zeta_{2}\right)$ of the form

$$
F\left(\zeta_{1}, \zeta_{2}\right)=\iint\left(t_{1}-\zeta_{1}\right)^{-1}\left(t_{2}-\zeta_{2}\right)^{-1}\left(t_{1}-t_{2}\right)^{2} \Phi\left(t_{1}, t_{2}\right) d t_{1} d t_{2}
$$

with $\Phi\left(t_{1}, t_{2}\right)$ bounded uniformly in each compact in $\mathbb{R}^{2}$ and such that uniformly in $\Im \zeta_{1,2} \geq 1$

$$
\left|F_{n}\left(\zeta_{1}, \zeta_{2}\right)-F\left(\zeta_{1}, \zeta_{2}\right)\right| \leq C\left(1+|\zeta|^{2}\right) n^{-1 / 4}
$$

Let also uniformly in $\operatorname{Im} \zeta \geq \varepsilon_{n}=(\log n)^{-1 / 2}$ and $\Re \zeta$ varying in any compact $L \subset \mathbb{R}$

$$
\left|F_{n}^{(1)}(\zeta)\right| \leq C(L)
$$

Then for any intervals $I_{1}, I_{2} \subset \mathbb{R}$

$$
\lim _{n \rightarrow \infty} \int_{I_{1}} d t_{1} \int_{I_{2}} d t_{2} \mathcal{K}_{n}^{2}\left(t_{1}, t_{2}\right)=\int_{I_{1}} d t_{1} \int_{I_{2}} d t_{2} \Phi\left(t_{1}, t_{2}\right)
$$

Proof of Proposition [5. Notice that

$$
F_{n}^{(1)}(\zeta)=n^{4 / 3} \delta_{n, n}(z)
$$

where $\delta_{k, n}(z)$ is defined in (2.43). Therefore, using the bound (2.56) in (2.41), we get for any $\Im \zeta \geq \varepsilon_{n}$

$$
\left|g_{n, n}\left(n^{-1 / 3} \zeta\right)+g_{n, n}\left(-n^{-1 / 3} \zeta\right)\right| \leq C n^{-2 / 3}\left(|\zeta|^{2}+1\right),
$$

where $C$ does not depend on $n$ and $\zeta$. On the other hand, taking $z=n^{-1 / 3}(a+i \varepsilon)$, we have for any $\epsilon>\epsilon_{n}$

$$
\begin{aligned}
& \int_{|t-a| \leq \varepsilon} \mathcal{K}_{n}(t, t) d t \leq 2 \varepsilon^{2} \int \frac{\mathcal{K}_{n}(t, t) d t}{(t-a)^{2}+\varepsilon^{2}}=2 \varepsilon n^{2 / 3} \Im g_{n, n}(z) \\
& \leq \varepsilon_{n} n^{2 / 3}\left(\Im g_{n, n}(z)+\Im g_{n, n}(-z)\right) \leq \varepsilon C\left(a^{2}+1\right)
\end{aligned}
$$

Take the integral

$$
\int_{\Im \zeta_{1}= \pm 1} d \zeta_{1} \int_{\Im \zeta_{2}= \pm 1} d \zeta_{2}\left(F_{n}\left(\zeta_{1}, \zeta_{2}\right)-F\left(\zeta_{1}, \zeta_{2}\right)\right) e^{-\left(\zeta_{1}-a_{1}\right)^{2} / 2 \sigma_{1}} e^{-\left(\zeta_{2}-a_{2}\right)^{2} / 2 \sigma_{2}} .
$$

Using the Cauchy theorem, we get that for any $\sigma_{1,2}>0, a_{1,2} \in \mathbb{R}$

$$
\left|\iint\left(t_{1}-t_{2}\right)^{2}\left(\mathcal{K}_{n}^{2}\left(t_{1}, t_{2}\right)-\Phi\left(t_{1}, t_{2}\right)\right) e^{-\left(t_{1}-a_{1}\right)^{2} / 2 \sigma_{1}} e^{-\left(t_{2}-a_{2}\right)^{2} / 2 \sigma_{2}} d t_{1} d t_{2}\right| \leq C n^{-1 / 4}
$$


with $C$, depending on $a_{1}, a_{2}, \sigma_{1}, \sigma_{2}$, but independent of $n$. This implies that for any Lipshitz $f_{1}$ and $f_{2}$ with a compact support

$$
\lim _{n \rightarrow \infty} \int\left(t_{1}-t_{2}\right)^{2}\left(\mathcal{K}_{n}^{2}\left(t_{1}, t_{2}\right)-\Phi\left(t_{1}, t_{2}\right)\right) f_{1}\left(t_{1}\right) f_{2}\left(t_{2}\right) d t_{1} d t_{2}=0
$$

For any small enough $\epsilon$ denote by $f_{1}^{(+\epsilon)}$ a Lipshitz function which coincides with the indicator $\chi_{I_{1}}$ of $I_{1}=\left(a_{1}, b_{1}\right)$ inside this interval, equals to zero outside of $\left(a_{1}-\epsilon, b_{1}+\epsilon\right)$ and is linear in $\left(a_{1}-\epsilon, a_{1}\right),\left(b_{1}, b_{1}+\epsilon\right)$. Let $f_{1}^{(-\epsilon)}$ be a similar function for the interval $\left(a_{1}+\epsilon, b_{1}-\epsilon\right)$ and $f_{2}^{( \pm \epsilon)}$ be similar functions for $I_{2}$. Denote also

$$
\phi_{\varepsilon_{1}}\left(t_{1}, t_{2}\right)=\left(t_{1}-t_{2}\right)^{-2} \mathbf{1}_{\left|t_{1}-t_{2}\right|>\varepsilon_{1}}+\varepsilon_{1}^{-2} \mathbf{1}_{\left|t_{1}-t_{2}\right| \leq \varepsilon_{1}} .
$$

Then, evidently

$$
\phi_{\varepsilon_{1}}\left(t_{1}, t_{2}\right) f_{1}^{(-\epsilon)}\left(t_{1}\right) f_{2}^{(-\epsilon)}\left(t_{2}\right) \leq \phi_{\varepsilon}\left(t_{1}, t_{2}\right) \chi_{I_{1}}\left(t_{1}\right) \chi_{I_{1}}\left(t_{2}\right) \leq \phi_{\varepsilon_{1}}\left(t_{1}, t_{2}\right) f_{1}^{(+\epsilon)}\left(t_{1}\right) f_{2}^{(+\epsilon)}\left(t_{2}\right) .
$$

Integrate this inequality with $\left(t_{1}-t_{2}\right)^{2} \mathcal{K}_{n}^{2}\left(t_{1}, t_{2}\right)$, and take the limits $n \rightarrow \infty$ and then $\epsilon \rightarrow 0$. We obtain

$$
\begin{aligned}
& \int_{I_{1} \times I_{2}} d t_{1} d t_{2} \Phi\left(t_{1}, t_{2}\right)-O\left(\varepsilon_{1}\right) \leq \lim _{n \rightarrow \infty} \int_{I_{1} \times I_{2}} d t_{1} d t_{2} \mathcal{K}_{n}^{2}\left(t_{1}, t_{2}\right) \\
& +\lim _{n \rightarrow \infty} \int_{I_{1} \times I_{2}} d t_{1} d t_{2} \mathcal{K}_{n}^{2}\left(t_{1}, t_{2}\right)\left(\varepsilon_{1}^{-2}\left(t_{1}-t_{2}\right)^{2}-1\right) \mathbf{1}_{\left|t_{1}-t_{2}\right| \leq \varepsilon_{1}} \leq \int_{I_{1} \times I_{2}} d t_{1} d t_{2} \Phi\left(t_{1}, t_{2}\right)+O\left(\varepsilon_{1}\right) .
\end{aligned}
$$

But using the inequality $\mathcal{K}_{n}^{2}\left(t_{1}, t_{2}\right) \leq \mathcal{K}_{n}\left(t_{1}, t_{1}\right) \mathcal{K}_{n}\left(t_{2}, t_{2}\right)$ and then (2.58), we obtain that the second limit is $O\left(\varepsilon_{1}\right)$. Then, taking the limit $\varepsilon_{1} \rightarrow 0$ we get the assertion of Proposition 5 ,

Let us check that in our case conditions (2.55) and (2.56) are satisfied. Using the ChristoffelDarboux formula, it is easy to derive from (2.53) that

$$
\begin{aligned}
F_{n}\left(\zeta_{1}, \zeta_{2}\right) & =\left(J_{n}^{(n)}\right)^{2} \int \frac{\left(\psi_{n}^{(n)}\left(\lambda_{1}\right) \psi_{n+1}^{(n)}\left(\lambda_{2}\right)-\psi_{n}^{(n)}\left(\lambda_{2}\right) \psi_{n+1}^{(n)}\left(\lambda_{1}\right)\right)^{2}}{\left(\lambda_{1}-z_{1}\right)\left(\lambda_{2}-z_{2}\right)} \lambda_{1} d \lambda_{2} \\
& =\left(J_{n}^{(n)}\right)^{2}\left[R_{n, n}\left(z_{1}\right) R_{n+1, n+1}\left(z_{2}\right)+R_{n, n}\left(z_{2}\right) R_{n+1, n+1}\left(z_{1}\right)\right. \\
F_{n}^{(1)}\left(\zeta_{1}\right) & =2\left(J_{n}^{(n)}\right)^{2}\left[R_{n, n}^{\prime}\left(z_{1}\right) R_{n+1, n+1}^{\prime}\left(z_{1}\right)-R_{n, n+1}^{\prime}\left(z_{1}\right) R_{n, n+1}^{\prime}\left(z_{1}\right)\right]
\end{aligned}
$$

where

$$
R_{k, m}(z)=\int \frac{\psi_{k}^{(n)}(\lambda) \psi_{m}^{(n)}(\lambda)}{z-\lambda} d \lambda
$$

is the resolvent of $J^{(n)}\left(R=\left(z-J^{(n)}\right)^{-1}\right)$.

Proposition 6. Let $\mathcal{J}$ be an arbitrary Jacobi matrix, with $\left|\mathcal{J}_{j, j+1}\right| \leq A$, for all $j$ such that $|j-k| \leq M$. Consider $\mathcal{R}(z)=(z-\mathcal{J})^{-1}$ with $|\Im z| \leq A_{1}$. Then

$$
\left|\mathcal{R}_{k, j}(z)\right| \leq \frac{C_{1}^{\prime}}{\Im z} e^{-C_{2}^{\prime}|\Im z||k-j|}+\frac{C_{1}^{\prime}}{|\Im z|^{2}} e^{-C_{2}^{\prime}|\Im z| M},
$$

where $C_{1}^{\prime}, C_{2}^{\prime}>0$ depend only on $A$ and $A_{1}$. 
Let us choose $N=\left[n^{1 / 3} \log ^{2} n\right]$. By (2.62), for $\alpha=0,1$ and $\Im z>n^{-1 / 3} \varepsilon_{n}$

$$
\sum_{k:|k-n|>N}\left|R_{n-\alpha, k}(z)\right|^{2} \leq C n e^{-C N \Im z} / \Im z \leq e^{-C_{1} \log ^{3 / 2} n} .
$$

Therefore for $\alpha, \beta=0,1$

$$
R_{n+\alpha, n+\beta}^{\prime}(z)=\sum_{|k|<N} R_{n+\alpha, k}(z) R_{n+\beta, k}(z)+O\left(e^{-C \log ^{3 / 2} n}\right) .
$$

Consider the matrix $\mathcal{J}^{(n, 2 N)}$ whose entries coincide with that of $\mathcal{J}^{(n)}$ with the only exception $J_{n \pm 2 N, n \pm 2 N+1}^{(n, 2 N)}=0$. Consider

$$
R^{(2 N)}(z)=\left(z-\mathcal{J}^{(n, 2 N)}\right)^{-1}
$$

We need also a simple observation, following from (2.62) and the resolvent identity

$$
R^{(1)}-R^{(0)}=R^{(0)}\left(M^{(1)}-M^{(0)}\right) R^{(1)}, \quad R^{(1,0)}=\left(z-M^{(1,0)}\right)^{-1} .
$$

Remark 7. If in the resolvent identity we take $M^{(0)}=\mathcal{J}^{(n)}$ and $M^{(1)}=\mathcal{J}^{(n, 2 N)}$ with $N_{k}=$ $|k|+n^{1 / 3} \log ^{2} n$, then (2.62) gives us for any $z:|\Im z|>n^{-1 / 3} \log ^{-1 / 2} n$ for any fixed $\delta$

$$
\left|R_{n+k, n+k+\delta}(z)-R_{n+k, n+k+\delta}^{(2 N)}\right| \leq C_{1}^{\prime} e^{-C_{2}^{\prime} \log ^{3 / 2} n} .
$$

Let us study first the case when in (1.16) $\tilde{s}=1$. Consider the Dirac operator $\mathcal{A}$ defined in $L_{2}(\mathbb{R}) \times L_{2}(\mathbb{R})$ by the differential expression (1.23)-(1.18). Let $\mathcal{R}_{\alpha, \beta}(x, y ; \zeta)(\alpha, \beta=0,1)$ be the kernel of the operator $\mathcal{R}(\zeta)=(\zeta-2 \mathcal{A})^{-1}$. It means that the coefficients $\mathcal{R}_{\alpha, \beta}(x, y ; \zeta)$ satisfy the equations

$$
\begin{aligned}
2 \frac{d}{d x} \mathcal{R}_{1,0}(x, y ; \zeta)+2 q(x) \mathcal{R}_{1,0}(x, y ; \zeta)-\zeta \mathcal{R}_{0,0}(x, y ; \zeta) & =\delta(x-y) \\
-2 \frac{d}{d x} \mathcal{R}_{0,1}(x, y ; \zeta)+2 q(x) \mathcal{R}_{0,1}(x, y ; \zeta)-\zeta \mathcal{R}_{1,1}(x, y ; \zeta) & =\delta(x-y) \\
-2 \frac{d}{d x} \mathcal{R}_{0,0}(x, y ; \zeta)+2 q(x) \mathcal{R}_{0,0}(x, y ; \zeta)-\zeta \mathcal{R}_{1,0}(x, y ; \zeta) & =0 \\
2 \frac{d}{d x} \mathcal{R}_{1,1}(x, y ; \zeta)+2 q(x) \mathcal{R}_{1,1}(x, y ; \zeta)-\zeta \mathcal{R}_{0,1}(x, y ; \zeta) & =0
\end{aligned}
$$

Here $\delta(x)$ is the Dirac $\delta$-function and, e.g., the first equation means that the l.h.s. is equal to zero, as $x \neq y$ and $2 \mathcal{R}_{1,0}(x+0, x)-2 \mathcal{R}_{1,0}(x-0, x)=1$.

Consider the $(4 N+1) \times(4 N+1)$ matrix with entries

$$
R_{n+2 k+\alpha, n+2 m+\beta}^{*}=(-1)^{(k+m)} \mathcal{R}_{\alpha, \beta}\left(\frac{2 k+\alpha}{n^{1 / 3}}, \frac{2 m+\beta}{n^{1 / 3}} ; \zeta\right),
$$

where $-N \leq k, m \leq N-1, \quad \alpha, \beta=0,1$. Let us prove, that for $|k|,|m| \leq N$

$$
\left|R_{n+k, n+m}^{(2 N)}\left(\zeta n^{-1 / 3}\right)-R_{n+k, n+m}^{*}\right| \leq C n^{-1 / 4}\left(1+|\zeta|^{2}\right) .
$$

Using equations (2.66), one can check that for all $-2 N \leq k, m \leq 2 N, \quad \alpha, \beta=0,1$

$$
\left(\left(z-J^{(n, 2 N)}\right) R^{*}\right)_{n+k, n+m}=\delta_{k, m}+d_{k, m}+a_{m}^{(1)} \delta_{k, 2 N}+a_{m}^{(2)} \delta_{k,-2 N}
$$


where the remainder terms could be estimated as follows:

$$
\begin{aligned}
\left|d_{k, m}\right| & \leq\left. C n^{-2 / 3} \sum_{\alpha, \beta=0,1}\left(1+|\zeta|^{2}+q^{2}(x)\right)\left|\mathcal{R}_{\alpha, \beta}(x, y ; \zeta)\right|\right|_{x=\frac{k+\alpha}{n^{1 / 3}, y=\frac{m+\theta(\alpha, \beta, k, m)}{n^{1 / 3}}}} \\
\left|d_{k, k}\right| & \leq\left. C n^{-1 / 3} \sum_{\alpha, \beta=0,1}(1+|\zeta|+q(x))\left|\mathcal{R}_{\alpha, \beta}(x, y ; \zeta)\right|\right|_{x=\frac{k+\alpha}{n^{1 / 3}, y=\frac{k+\theta(\alpha, \beta, k, k)}{n^{1 / 3}}}}
\end{aligned}
$$

with $\left|\theta_{\alpha, \beta, k, m}\right| \leq 2$. The components of the vectors $\mathbf{a}^{(1)}$ and $\mathbf{a}^{(2)}$ satisfy the bounds

$$
\begin{aligned}
& \left|a_{2 m+\alpha}^{(1)}\right| \leq\left|\mathcal{R}_{0, \alpha}\left((2 N+1) n^{-1 / 3},(2 m+\alpha) n^{-1 / 3} ; \zeta\right)\right|+C n^{-1 / 3}\left(|\zeta|+\left|\log ^{2} n\right|\right) \\
& \left|a_{2 m+\alpha}^{(2)}\right| \leq\left|\mathcal{R}_{0, \alpha}\left((-2 N-1) n^{-1 / 3},(2 m+\alpha) n^{-1 / 3} ; \zeta\right)\right|+C n^{-1 / 3}\left(|\zeta|+\left|\log ^{2} n\right|\right) .
\end{aligned}
$$

Let us define the $(4 N+1) \times(4 N+1)$ matrices

$$
D_{n+i, n+j}=d_{n+i, n+j}, \quad \tilde{D}_{n+i, n+j}=a_{j}^{(1)} \delta_{i, 2 N}+a_{j}^{(2)} \delta_{i,-2 N} .
$$

Then (2.69) can be rewritten as

$$
\left(z-J^{(n, 2 N)}\right) R^{*}=I+D+\tilde{D} .
$$

Using the trivial bound for the norm of any matrix $\|A\|^{2} \leq \operatorname{Tr} A A^{*}$ and the bound for the resolvent of the Dirac operator (see [14])

$$
\int\left|\mathcal{R}_{\alpha, \beta}(x, y ; \zeta)\right|^{2} d y \leq C|\Im \zeta|^{-2}(|q(x)|+|\zeta|),
$$

we obtain from the first and the second line of (2.70) that for $|\Im \zeta|>\varepsilon_{n}$

$$
\|D\| \leq C(|\zeta|+1) n^{-1 / 3} \log ^{3} n \leq C(|\zeta|+1) n^{-1 / 4} .
$$

On the other hand, it follows from (2.72) that for any $-2 N \leq k, m \leq 2 N$

$$
R_{n+k, n+m}^{(2 N)}=\left(z-J^{(n, 2 N)}\right)_{n+k, n+m}^{-1}=\left(R^{*}(I+D+\tilde{D})^{-1}\right)_{n+k, n+m}
$$

and so we can write

$$
\left|R_{n, n}^{(2 N)}-\left(R^{*}(I+\tilde{D})^{-1}\right)_{n, n}\right| \leq\|D\| \cdot\left\|R^{*}\right\| \frac{\left\|(I+\tilde{D})^{-2}\right\|}{1-\|D\|||(I+\tilde{D})^{-2} \|} .
$$

But for any $\mathbf{x} \in \mathbb{R}^{4 N+1}$,

$$
(I+\tilde{D}) \mathbf{x}=\mathbf{x}+\mathbf{e}_{n+2 N}\left(\mathbf{x}, \mathbf{a}^{(1)}\right)+\mathbf{e}_{n-2 N}\left(\mathbf{x}, \mathbf{a}^{(2)}\right),
$$

where $\left\{\mathbf{e}_{k}\right\}_{k=n-2 N}^{n+2 N}$ is a standard basis in $\mathbb{R}^{4 N+1}$. So we get

$$
\left\|(I+\tilde{D})^{-1}\right\| \leq\left\|A^{-1}\right\|+1, \quad A=\left(\begin{array}{cc}
1+a_{n+2 N-1}^{(1)} & a_{n-2 N}^{(1)} \\
a_{n+2 N-1}^{(2)} & 1+a_{n-2 N}^{(2)}
\end{array}\right) .
$$

The off diagonal entries of $A$ tend to zero, because they are expressed in terms of the resolvent $\mathcal{R}_{\alpha, \beta}(x, y ; \zeta)$ with $|x-y| \sim \log ^{2} n$ (see (2.71)). $a_{n-2 N-1}^{(1)} \rightarrow 0$, because $q(x) \sim C \sqrt{-x}$, as $x \rightarrow-\infty$. And $\left|a_{n+2 N}^{(2)}\right| \rightarrow 1 / 4$, because if the potential tends to zero fast enough, then the 
resolvent near the diagonal coincides asymptotically with that for $q=0$ (see [14]). Hence, using (2.75) and (2.77), we get

$$
\left|R_{k, m}^{(2 N)}-\left(R^{*}(I+\tilde{D})^{-1}\right)_{k, m}\right| \leq C n^{-1 / 4}\left(1+|\zeta|^{2}\right) .
$$

But from (2.76) we derive

$$
(I+\tilde{D})_{k, m}^{-1}=\delta_{k, m}, \quad k \neq \pm 2 N .
$$

Hence, we obtain from (2.78) and (2.62)

$$
\left|R_{n, n}^{(2 N)}-R_{n, n}^{*}\right| \leq C n^{-1 / 4}+\|(I+\tilde{D})^{-1}||\left(\left|R_{n, 2 N}^{*}\right|+\left|R_{n,-2 N}^{*}\right|\right) \leq C n^{-1 / 4}
$$

and we have proved (2.55) with

$$
\begin{aligned}
F\left(\zeta_{1}, \zeta_{2}\right)=\left(\mathcal{R}_{0,0}\left(0,0 ; \zeta_{1}\right) \mathcal{R}_{1,1}\left(0,0 ; \zeta_{2}\right)+\right. & \mathcal{R}_{0,0}\left(0,0 ; \zeta_{2}\right) \mathcal{R}_{1,1}\left(0,0, \zeta_{1}\right) \\
& \left.-2 \mathcal{R}_{0,1}\left(0,0+0 ; \zeta_{1}\right) \mathcal{R}_{1,0}\left(0+0,0 ; \zeta_{2}\right)\right)
\end{aligned}
$$

where we denote $\mathcal{R}_{0,1}\left(0,0+0, \zeta_{1}\right)=\lim _{x \rightarrow+0} \mathcal{R}_{0,1}\left(0, x, \zeta_{1}\right)$. But according to the spectral theorem (see [14]),

$$
\mathcal{R}_{\alpha, \beta}(x, y ; \zeta)=\int \frac{\Psi_{\alpha}(x ; t) \Psi_{\beta}(y ; t)}{\zeta-2 t} d t
$$

where $\boldsymbol{\Psi}(x ; t)=\left(\Psi_{0}(0 ; t), \Psi_{1}(0 ; t)\right)$ is the solution of the Dirac system (1.23), satisfying asymptotic conditions (1.24). The last two relations and the formula of the inverse Stieltjes transform yield

$$
\Phi\left(t_{1}, t_{2}\right)=(2 \pi)^{-2}\left(\Psi_{1}\left(0 ; t_{1} / 2\right) \Psi_{0}\left(0 ; t_{2} / 2\right)-\Psi_{0}\left(0 ; t_{2} / 2\right) \Psi_{1}(0 ; t / 2)\right)^{2} .
$$

Moreover, similarly to (2.79) we obtain

$$
\begin{aligned}
n^{-1 / 3} \sum_{|k-n| \leq N}\left(R_{n, k}^{(2 N)}\right)^{2} \leq \int \mathcal{R}_{0,0}^{2}(0, y ; \zeta) d y+\int & \mathcal{R}_{0,1}^{2}(0, y ; \zeta) d y+O\left(\|D\| \cdot|\Im \zeta|^{-2}\right) \\
& =-\frac{\partial}{\partial \zeta} \mathcal{R}_{0,0}(0,0 ; \zeta)+O\left(\|D\| \cdot|\Im \zeta|^{-2}\right)
\end{aligned}
$$

Using the representation (2.81) and taking into account that $\Psi_{\alpha}(x ; t)$ are smooth function with respect to $t$, according to the standard theory of the Cauchy type integrals (see [16]) we get that the derivative in the r.h.s. of (2.83) is uniformly bounded up to the real line. Therefore we obtain (2.56) and then, on the basis of Proposition (5), obtain the assertion of Theorem 2 for $l=2$. For the other $l$ we study by the same way

$$
F_{n}\left(\zeta_{1}, \ldots, \zeta_{l}\right)=\int \prod_{i=1}^{l}\left(t_{i}-\zeta_{i}\right)^{-1}\left(t_{1}-t_{2}\right) \ldots\left(t_{l}-t_{1}\right) \mathcal{K}_{n}\left(t_{1}, t_{2}\right) \ldots \mathcal{K}_{n}\left(t_{l}, t_{1}\right) d t_{1} \ldots d t_{l}
$$

Now, notice that the $\left(\Psi_{0}(x, t), \Psi_{1}(x, t)\right) \rightarrow\left(-\Psi_{1}(x, t), \Psi_{0}(x, t)\right)$ gives us the solution of (1.23) with potential $q_{1}(x)=-q(x)$ but does not change the expression (1.22). This completes the proof of Theorem 2

To prove Corollary 1 we split the expansion for the Fredholm determinant in two parts: with $m<N$ and $m \geq N$ ( $m$ is the number of variables in the correspondent determinant). Using the Hadamard bound for determinants with $m>N$ and then (2.58) it is easy to see that the second sum possesses the bound $C^{N} / N$ !. Hence using Theorem 2 we can take the limit $n \rightarrow \infty$ in the first sum and then take the limit $N \rightarrow \infty$. Relation (1.25) follows. 


\section{Auxiliary results}

Proof of Lemma 1. We introduce an eigenvalue distribution which is more general than (1.4), making different the number of variable and the large parameter in front of $V$ in the exponent of the r.h.s of (1.4):

$$
p_{k, n}\left(\lambda_{1}, \ldots \lambda_{k}\right)=Z_{k, n}^{-1} \prod_{1 \leq j<m \leq k}\left(\lambda_{j}-\lambda_{m}\right)^{2} \exp \prod_{j=1}^{k} e^{-n V\left(\lambda_{j}\right)},
$$

where $Z_{k, n}$ is the normalizing factor. For $k=n$ this probability distribution density coincides with (1.4). Let

$$
\tilde{\rho}_{k, n}\left(\lambda_{1}\right)=\int d \lambda_{2} \ldots d \lambda_{k} p_{k, n}\left(\lambda_{1}, \ldots \lambda_{k}\right), \quad \tilde{\rho}_{k, n}\left(\lambda_{1}, \lambda_{2}\right)=\int d \lambda_{3} \ldots d \lambda_{k} p_{k, n}\left(\lambda_{1}, \ldots \lambda_{k}\right)
$$

be the first and the second marginal densities of (3.1). By the standard argument [15] we obtain

$$
\begin{aligned}
& \tilde{\rho}_{k, n}(\lambda)=k^{-1} K_{k, n}(\lambda, \lambda), \\
& \tilde{\rho}_{k, n}(\lambda, \mu)=\frac{1}{k(k-1)}\left[K_{k, n}(\lambda, \lambda) K_{k, n}(\mu, \mu)-K_{k, n}^{2}(\lambda, \mu)\right],
\end{aligned}
$$

where $K_{k, n}(\lambda, \mu)$ is defined in (2.40). Remark also that

$$
\tilde{\rho}_{k, n}(\lambda)=\frac{n}{k} \rho_{k, n}(\lambda)
$$

where $\rho_{k, n}$ is defined in (2.40). Taking any twice differentiable and vanishing outside $\sigma_{2 \varepsilon}$ function $\phi(\lambda)$ and integrating by parts with respect to $V$, we come to the identity

$$
\int V^{\prime}(\lambda) \tilde{\rho}_{k, n}(\lambda) \phi(\lambda) d \lambda=\frac{1}{n} \int \tilde{\rho}_{k, n}(\lambda) \phi^{\prime}(\lambda) d \lambda+2 \frac{k-1}{n} \int \tilde{\rho}_{k, n}(\lambda, \mu) \frac{\phi(\lambda)}{\lambda-\mu} d \lambda d \mu .
$$

The symmetry property $\tilde{\rho}_{k, n}(\lambda, \mu)=\tilde{\rho}_{k, n}(\mu, \lambda)$ of (3.2) implies

$$
\int \tilde{\rho}_{k, n}(\lambda, \mu) \frac{\phi(\lambda)}{\lambda-\mu} d \lambda d \mu=-\int \tilde{\rho}_{k, n}(\lambda, \mu) \frac{\phi(\mu)}{\lambda-\mu} d \lambda d \mu .
$$

This allows us to rewrite (3.4) in the form

$$
\int V^{\prime}(\lambda) \tilde{\rho}_{k, n}(\lambda) \phi(\lambda) d \lambda=\frac{1}{n} \int \tilde{\rho}_{k, n}(\lambda) \phi^{\prime}(\lambda) d \lambda+\frac{k-1}{n} \int \tilde{\rho}_{k, n}(\lambda, \mu) \frac{\phi(\lambda)-\phi(\mu)}{\lambda-\mu} d \lambda d \mu .
$$

Now, using (3.3) and the fact that

$$
\int K_{k, n}^{2}(\lambda, \mu) d \mu=K_{k, n}(\lambda, \lambda)
$$

we can rewrite the last equation as

$$
\int \frac{\phi(\lambda)-\phi(\mu)}{\lambda-\mu} \rho_{k, n}(\lambda) \rho_{k, n}(\mu) d \lambda d \mu-\int V^{\prime}(\lambda) \rho_{k, n}(\lambda) \phi(\lambda) d \lambda+\delta_{k, n}(\phi)=0
$$

where we denote

$$
\delta_{k, n}(\phi)=\frac{1}{2 n^{2}} \int\left(\phi^{\prime}(\lambda)+\phi^{\prime}(\mu)-2 \frac{\phi(\lambda)-\phi(\mu)}{\lambda-\mu}\right) K_{k, n}^{2}(\lambda, \mu) d \lambda d \mu .
$$


Subtracting from (3.5) the relation obtained from (3.5) by the replacement $k \rightarrow(k-1)$ and multiplying the difference by $n$, we obtain:

$$
\begin{aligned}
2 \int \frac{\phi(\lambda)-\phi(\mu)}{\lambda-\mu} \rho(\mu)\left[\psi_{k}^{(n)}(\lambda)\right]^{2} d \lambda d \mu-\int V^{\prime}(\lambda) \phi(\lambda)\left[\psi_{k}^{(n)}(\lambda)\right]^{2} d \lambda & \\
& +\delta_{k, n}^{(R)}(\phi)+\tilde{\delta}_{k, n}^{(R)}(\phi)=0
\end{aligned}
$$

where

$$
\begin{aligned}
& \delta_{k, n}^{(R)}(\phi)=\frac{1}{n} \int K_{k, n}(\lambda, \mu) \psi_{k}^{(n)}(\lambda) \psi_{k}^{(n)}(\mu)\left(\phi^{\prime}(\lambda)+\phi^{\prime}(\mu)-2 \frac{\phi(\lambda)-\phi(\mu)}{\lambda-\mu}\right) d \lambda d \mu \\
& \tilde{\delta}_{k, n}^{(R)}(\phi)=\int \frac{\phi(\lambda)-\phi(\mu)}{\lambda-\mu}\left(\rho_{k, n}(\mu)-\rho(\mu)\right)\left[\psi_{k}^{(n)}(\lambda)\right]^{2} d \lambda d \mu-\frac{1}{n} \int \phi^{\prime}(\lambda)\left[\psi_{k}^{(n)}(\lambda)\right]^{2} d \lambda .
\end{aligned}
$$

By Schwartz inequality

$$
\begin{aligned}
&\left|\delta_{k, n}^{(R)}(\phi)\right| \leq \frac{2}{n}\left\|\phi^{\prime \prime}\right\|_{0}\left(\int K_{k, n}^{2}(\lambda, \mu)(\lambda-\mu)^{2} d \lambda d \mu\right)^{1 / 2} \\
& \cdot\left(\int\left(\psi_{k}^{(n)}(\lambda)\right)^{2}\left(\psi_{k}^{(n)}(\mu)\right)^{2} d \lambda d \mu\right)^{1 / 2} \leq \frac{C}{n}\left\|\phi^{\prime \prime}\right\|_{0} \\
&\left|\tilde{\delta}_{k, n}^{(R)}(\phi)\right| \leq\left|\tilde{\delta}_{0, n}^{(R)}(\phi)\right|+\frac{|k-n|}{n}\left\|\phi^{\prime}\right\|_{0} \leq C\left(\left\|\phi^{\prime}\right\|_{2}^{1 / 2}\left\|\phi^{\prime \prime}\right\|_{2}^{1 / 2} \frac{\log ^{1 / 2} n}{n^{1 / 2}}+\left\|\phi^{\prime}\right\|_{0} \frac{|k-n|}{n}\right),
\end{aligned}
$$

where the symbols $\|\ldots\|_{0}$ and $\|\ldots\|_{2}$ denotes the supremum and the $L_{2}$-norm on $\sigma_{\varepsilon}$. Here we have used the result of [5], valid for any smooth function $\phi(\mu)$ defined on $\sigma_{\varepsilon}$

$$
\left|\int \phi(\mu) \rho_{n, n}(\mu) d \mu-\int \phi(\mu) \rho(\mu) d \mu\right| \leq C\left\|\phi^{\prime}\right\|_{2}^{1 / 2}\|\phi\|_{2}^{1 / 2} n^{-1 / 2} \log ^{1 / 2} n
$$

where the symbol $\|\ldots\|_{2}$ denotes the $L_{2}$-norm on $\sigma_{\varepsilon}$.

Now we are going to use (1.3) in the second integral in the r.h.s. of (3.7). But since this representation is valid only for $\lambda \in[-2,2]$ we need to restrict the integrals in (3.7) by some $\sigma_{\tilde{\varepsilon}}=[-2-\tilde{\varepsilon}, 2+\tilde{\varepsilon}]$ with some small $\tilde{\varepsilon}>0$. To this aim we use

Proposition 7. Consider any unitary invariant ensemble of the form (1.1) and assume that $V(\lambda)$ possess two bounded derivatives in some neighborhood of the support $\sigma$ of the density of states $\rho$. Let also $\sigma$ consist of a finite number of intervals, $\rho(\lambda)$ satisfy condition $C 4$ and $\rho(\lambda) \sim C\left(a^{*}\right)\left|\lambda-a^{*}\right|^{1 / 2}$ near any edge point $a^{*}$ of $\sigma$.

Then there exist absolute constants $C, C_{0}, \varepsilon_{0}>0$ such that for any positive $C_{0} n^{-1 / 2} \log n \leq$ $\varepsilon \leq \varepsilon_{0}$ and for any integer $k:|k| \leq n+n^{1 / 2}$ the bounds hold:

$$
\int_{\mathbf{R} \backslash \sigma_{\varepsilon}} \rho_{k, n}(\lambda) d \lambda \leq e^{-n C \varepsilon}, \quad \int_{\mathbf{R} \backslash \sigma_{\varepsilon}}\left(\psi_{k}^{(n)}(\lambda)\right)^{2} d \lambda \leq e^{-n C \varepsilon} .
$$

This proposition was proved in [3]. It allows us to restrict the integration in the first three integrals of (3.7) by $\sigma_{\tilde{\varepsilon}}$ with $\tilde{\varepsilon}=C_{0} n^{-1 / 2} \log n$. Now we can use (1.3). The error, which appear because of this replacement is of the order $O(\tilde{\varepsilon})$, because $V^{\prime}(\lambda)$ is a smooth function in $\sigma_{\tilde{\varepsilon}}$. Hence, (3.7) can be rewritten in the form

$$
2 \int_{\sigma_{\tilde{\varepsilon}}}\left(\psi_{k}^{(n)}(\lambda)\right)^{2} d \lambda \int_{-2}^{2} \frac{\phi(\mu)}{\lambda-\mu} \rho(\mu) d \mu=\delta_{k, n}^{(R)}(\phi)+\tilde{\delta}_{k, n}^{(R)}(\phi)+O\left(\|\phi\|_{0} n^{-1 / 2} \log n\right) .
$$


Take $\phi(\lambda)=P_{0}^{-1}(\lambda)(z-\lambda)^{-1}$ and substitute in (3.12). Then, according to (1.13), we get

$$
2 \int_{\sigma_{\tilde{\varepsilon}}}\left(\psi_{k}^{(n)}(\lambda)\right)^{2} d \lambda \int_{-2}^{2} \frac{\mu^{2} \sqrt{4-\mu^{2}}}{(z-\mu)(\lambda-\mu)} d \mu=\delta_{k, n}^{(R)}(z)+\tilde{\delta}_{k, n}^{(R)}(z)+O\left(|\Im z|^{-1} n^{-1 / 2} \log n\right),
$$

where $\delta_{k, n}^{(R)}(z)$ and $\tilde{\delta}_{k, n}^{(R)}(z)$ have the form (3.8) and due to (3.9) satisfy the bound

$$
\left|\delta_{k, n}^{(R)}(z)\right| \leq \frac{C}{n|\Im z|^{2}}, \quad\left|\tilde{\delta}_{k, n}^{(R)}(z)\right| \leq \frac{C k}{n|\Im z|^{2}}+\frac{C \log ^{1 / 2} n}{n^{1 / 2}|\Im z|^{2}} .
$$

Thus, using the fact that

$$
\frac{2}{\pi} \int \frac{\mu^{2} \sqrt{4-\mu^{2}}}{(z-\mu)(\lambda-\mu)} d \mu=\frac{z^{2} \sqrt{z^{2}-4}}{z-\lambda}-\left(z^{2}+z \lambda+\lambda^{2}\right)+2,
$$

we get from (3.13)

$$
R_{k, k}(z)=\left(z^{2}+a_{k}+\delta_{k, n}^{(R)}(z)+\tilde{\delta}_{k, n}^{(R)}(z)+O\left(|\Im z|^{-1} n^{-1 / 2} \log n\right)\right) \frac{1}{z^{2} \sqrt{z^{2}-4}},
$$

where $R_{k, k}(z)$ is defined in (2.61) and we denote

$$
a_{k}=\int \lambda^{2}\left[\psi_{k}^{(n)}(\lambda)\right]^{2} d \lambda-2 .
$$

Let us assume that $a_{k}>C n^{-1 / 2} \log ^{1 / 2} n$ with $C$ big enough. Then, using the bound (3.14) and the Rouchet theorem, we get that $R_{k, k}(z)$ has a root in the circle of radius $\frac{1}{2} a_{k}^{1 / 2}$ centered in the point $i a_{k}^{1 / 2}$. But, by definition (2.61),

$$
\Im R_{k, k}(z) \Im z<0
$$

so $R_{k, k}(z)$ cannot have zeros, when $\Im z \neq 0$ and therefore we get $a_{k} \leq C n^{-1 / 4} \log ^{1 / 2} n$. Similarly, if we assume that $a_{k} \leq-C n^{-1 / 4} \log ^{1 / 2} n$ we get that $\Im R_{k, k}\left(\frac{1}{2}\left|a_{k}\right|^{1 / 2} e^{i \pi / 6}\right)>0$, which also contradict to (3.17). Thus, we obtain that

$$
\left|a_{k}\right| \leq C n^{-1 / 4} \log ^{1 / 2} n \text {. }
$$

From (3.16) and (3.15) we find

$$
\begin{aligned}
\left(J_{k}^{(n)}\right)^{2}+\left(J_{k-1}^{(n)}\right)^{2} & =\int_{\sigma_{e}} \lambda^{2}\left(\psi_{k}^{(n)}(\lambda)\right)^{2} d \lambda=2+a_{k} \\
\left(\left(J_{k}^{(n)}\right)^{2}+\left(J_{k-1}^{(n)}\right)^{2}\right)^{2}+\left(J_{k}^{(n)}\right)^{2}\left(J_{k+1}^{(n)}\right)^{2} & \\
+\left(J_{k-1}^{(n)}\right)^{2}\left(J_{k-2}^{(n)}\right)^{2} & =\int_{\sigma_{e}} \lambda^{4}\left(\psi_{k}^{(n)}(\lambda)\right)^{2} d \lambda=\frac{1}{2 \pi i} \oint_{L} \zeta^{4} R_{k, k}(\zeta) d \zeta \\
& =6+2 a_{k}+O\left(n^{-1 / 2} \log n\right) .
\end{aligned}
$$

Using here the first equation for $k:=k \pm 1$ to express $\left(J_{k \pm 1}^{(n)}\right)^{2}$ and $\left(J_{k-2}^{(n)}\right)^{2}$ through $\left(J_{k}^{(n)}\right)^{2}$, we obtain

$$
J_{k}^{2}=1+\frac{a_{k}}{2}+\frac{a_{k+1}-a_{k-1}}{4} \pm\left[\frac{a_{k+1}+2 a_{k}+a_{k-1}}{2}+O\left(n^{-1 / 2} \log n\right)\right]^{1 / 2} .
$$

Combining this relation with (3.18), we get the first statement of Lemma 1. The second statement follows from the first one and the first equation of (3.19). 


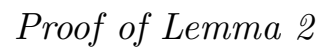

Choose $M=c n^{1 / 3}$, where the constant $c$ is small enough to provide the condition

$$
d C_{2} c<C_{1} / 7
$$

where $C_{1}$ and $C_{2}$ are the constants from (4.5) and $d=\pi(2+\varepsilon)^{-1}$. This condition and (4.5) guarantee that for any $l, l^{\prime}:\left|l-l^{\prime}\right|>n^{1 / 3} / 6$ and any $j:|j|<M,|t| \leq 1$

$$
\left|\left(e^{i t d j \mathcal{J}^{(0)}}\right)_{l, l^{\prime}}\right| \leq C e^{d C_{2} M-C_{1}\left|l-l^{\prime}\right| / 4} \leq C e^{-C_{1} n^{1 / 3} / 42} .
$$

Applying (4.3) three times we get (2.6) with

$$
\begin{aligned}
& \mathcal{P}_{k-l}^{(\delta)}=c_{1}^{(\delta)} \delta_{k, l}+\int_{s_{1}+s_{2}=1} d s_{1} d s_{2} \sum_{j=\infty}^{\infty} v_{j}(i j d)\left(e^{i j d s_{1} \mathcal{J}^{(0)}} E^{(l)} e^{i j d s_{2} \mathcal{J}^{(0)}}\right)_{k, k+\delta}, \\
& \mathcal{P}_{l_{1}, l_{2}}^{(2, k, \delta)}=\int_{\sum s_{i}=1} d s_{1} d s_{2} d s_{3} \sum_{j=-M}^{M} v_{j}(i j d)^{2}\left(e^{i j d s_{1} \mathcal{J}^{(0)}} E^{\left(l_{1}\right)} e^{i j d s_{2} \mathcal{J}^{(0)}} E^{\left(l_{2}\right)} e^{i j d s_{3} \mathcal{J}^{(0)}}\right)_{k, k+\delta}, \\
& \mathcal{P}_{l_{1}, l_{2}, l_{3}}^{(3, k, \delta)}=\int_{\sum s_{i}=1} d s_{1} \ldots d s_{4} \sum_{j=-M}^{M} v_{j}(i j d)^{3} \\
& \left(e^{i j d s_{1} \mathcal{J}^{(0)}} E^{\left(l_{1}\right)} e^{i j d s_{2} \mathcal{J}^{(0)}} E^{\left(l_{2}\right)} e^{i j d s_{3} \mathcal{J}^{(0)}} E^{\left(l_{3}\right)} e^{i j d s_{4} \mathcal{J}^{(0)}}\right)_{k, k+\delta}, \\
& r_{k}^{(\delta)}=\sum_{l_{1}, \ldots, l_{4}} \int_{\sum s_{i}=1} d s_{1} \ldots d s_{5} \sum_{j=-M}^{M} v_{j}(i j d)^{4} \\
& \left(e^{i j d s_{1} \mathcal{J}^{(0)}} \tilde{\mathcal{J}} e^{i j d s_{2} \mathcal{J}^{(0)}} \tilde{\mathcal{J}} e^{i j d s_{3} \mathcal{J}^{(0)}} \tilde{\mathcal{J}} e^{i j d s_{4} \mathcal{J}^{(0)}} \tilde{\mathcal{J}} e^{i j d s_{5}\left(\mathcal{J}^{(0)}+\tilde{\mathcal{J}}\right)}\right)_{k, k+\delta} \\
& +\int_{s_{1}+s_{2}=1} d s_{1} d s_{2} \sum_{|j|>M} v_{j}(i j d)\left(e^{i j d s_{1} \mathcal{J}^{(0)}} \tilde{J}\left(e^{i j d s_{2} \mathcal{J}^{(0)}}-e^{i j d s_{2}\left(\mathcal{J}^{(0)}+\tilde{\mathcal{J}}\right)}\right)\right)_{k, k+\delta},
\end{aligned}
$$

where we denote by $E^{(l)}$ a matrix with entries:

$$
E_{k, m}^{(l)}=\delta_{k, l} \delta_{m, l+1}+\delta_{k, l+1} \delta_{m, l} .
$$

Using the Schwartz inequality, we have

$$
\sum_{j}|j|^{4}\left|v_{j}\right| \leq\left(\sum_{j}|j|^{10}\left|v_{j}\right|^{2}\right)^{1 / 2}\left(\sum_{j \neq 0}|j|^{-2}\right)^{1 / 2} \leq C,
$$

Hence, using again the Schwartz inequality, we obtain

$$
\left|r_{k}^{(\delta)}\right| \leq m_{k}^{4} d^{4} \sum_{|j|<M}|j|^{4}\left|v_{j}\right|+m_{k} d \sum_{|j|>M}|j|\left|v_{j}\right| \leq C m_{k}^{4}+C m_{k} M^{-7 / 2} \leq C m_{k}^{4}
$$

where the last inequality is valid because of the choice of $M$ and (2.5).

To obtain (2.7) we use the representation (see [1]):

$$
\left(e^{i j d s \mathcal{J}^{(0)}}\right)_{k, l}=\frac{1}{2 \pi} \int_{-\pi}^{\pi} e^{i j d s \cos x} e^{i(k-l) x} d x=\mathbf{J}_{k-l}(j d s),
$$


where $\mathbf{J}_{k}(s)$ is the Bessel function. But it is well known (see, e.g. [1]) that the Bessel functions satisfy the following recurrent relations:

$$
k \mathbf{J}_{k}(s)=\frac{s}{2}\left(\mathbf{J}_{k+1}(s)+\mathbf{J}_{k-1}(s)\right) .
$$

Thus, e.g., the first sum in (2.7) can be expressed via the terms

$$
\begin{aligned}
& \sum_{|j|<M} v_{j}(i j d)^{2} \int_{\sum s_{i}=1} d s_{1} d s_{2} d s_{3}\left(d j s_{1}+\alpha_{1}\right)\left(d j s_{1}+\alpha_{2}\right) \\
& \cdot \sum^{\prime} \mathbf{J}_{k-l_{1}+\alpha_{3}}\left(d j s_{1}\right) \tilde{x}_{l_{1}} \mathbf{J}_{l_{1}-l_{2}+\alpha_{4}}\left(d j s_{2}\right) \tilde{y}_{l_{2}} \mathbf{J}_{l_{2}-k+\alpha_{5}}\left(2 j s_{3}\right),
\end{aligned}
$$

where $\alpha_{1}, \ldots \alpha_{5}$ can take the values $0, \pm 1, \pm(\delta+1)$. It is easy to see that any of these sums can be written in the form:

$$
\left(e^{i j d s_{2} \mathcal{J}^{(0)}} X^{\left(\alpha_{3}\right)} e^{i j d s_{2} \mathcal{J}^{(0)}} Y^{\left(\alpha_{4}\right)} e^{i j d s_{2} \mathcal{J}^{(0)}}\right)_{k, k+\alpha_{5}}, \quad X_{k, l}^{(\alpha)}=\delta_{k, \alpha+l} \tilde{x}_{k}, \quad Y_{k, l}^{(\alpha)}=\delta_{k, \alpha+l} \tilde{y}_{k},
$$

where evidently

$$
\left\|X^{(\alpha)}\right\| \leq \max \left|\tilde{x}_{k}\right|, \quad\left\|Y^{(\alpha)}\right\| \leq \max \left|\tilde{y}_{k}\right| .
$$

Hence, similarly to (3.24) we obtain

$$
\left|\sum^{\prime} \mathcal{P}_{l_{1}, l_{2}}^{(2, k, \delta)}\left(l_{1}-k\right)^{2} \tilde{x}_{l_{1}} \tilde{y}_{l_{2}}\right| \leq C\|\tilde{x}\|\|\tilde{y}\| \sum_{|j|<M}|j|^{4}\left|v_{j}\right| \leq C\|\tilde{x}\|\|\tilde{y}\| .
$$

The other inequalities in (2.7) can be proved similarly.

We are left to prove (2.9). Due to representations (3.23) and (3.25), we derive that $\mathcal{P}^{(\delta)}$ can be represented in the form (2.8) with

$$
F^{(\delta)}(x)=\sum \mathcal{P}_{l}^{(\delta)} e^{i l x}
$$

Using (3.23) and (3.25) we get

$$
\begin{aligned}
F^{(1)}(x)= & c_{1}^{(1)}+\sum_{j}(i j d) v_{j} \int_{0}^{1} d s_{1} \sum_{l} \frac{1}{4 \pi^{2}} \int_{-\pi}^{\pi} \int_{-\pi}^{\pi} e^{i l\left(-x_{1}+x_{2}+x\right)}\left(1+e^{-i\left(x_{1}+x_{2}\right)}\right) \\
& \cdot \exp \left\{2 i j d\left[s_{1} \cos x_{1}+\left(1-s_{1}\right) \cos x_{2}\right]\right\} d x_{1} d x_{2} \\
= & c_{1}^{(1)}+\frac{1}{2 \pi} \int_{-\pi}^{\pi} \frac{v\left(2 \cos x_{1}\right)-v\left(2 \cos \left(x_{1}-x\right)\right)}{\cos x_{1}-\cos \left(x_{1}-x\right)}\left(1+\cos \left(2 x_{1}-x\right)\right) d x_{1} \\
= & c_{1}^{(1)}+\frac{1}{2 \pi} \int_{-\pi}^{\pi} v\left(2 \cos x_{1}\right)\left(\frac{1+\cos \left(2 x_{1}-x\right)}{\cos x_{1}-\cos \left(x_{1}-x\right)}+\frac{1+\cos \left(2 x_{1}+x\right)}{\cos x_{1}-\cos \left(x_{1}+x\right)}\right) d x_{1} \\
= & P(2 \cos (x / 2))+P(-2 \cos (x / 2)) .
\end{aligned}
$$

Representation (2.10) can be obtained similarly. Lemma 2 is proven.

Proof of Proposition Q 2. Let us remark first that all limiting expression in the r.h.s. of (2.19) and (2.20) correspond to infinite sums over $j$ in the definitions (3.23) and infinite sums with respect to all $l_{i}$. The estimates for the remainder terms, which appears because of the restriction of summation in (3.23) over $|j|<M$, were obtained already in the proof of Lemma 2. And the remainders, which appear because of the replacement of infinite sums by sums over $\left|l_{i}\right|<N_{k}$, can be estimated by $O\left(e^{-C_{1} n^{1 / 3} / 12}\right)$ due to (3.22). Thus we are left to compute infinite over $l_{i}$ sums for $\mathcal{P}_{l_{1}, l_{2}}^{(2, k, \delta)}$ and $\mathcal{P}_{l_{1}, l_{2}, l_{3}}^{(3, k, \delta)}$ 
The first relation in (2.19) follows immediately from (2.8) and (3.26). To obtain the others let us consider an infinite Jacobi matrix $\mathcal{J}^{(\pi)}$ with $J_{k, k+1}^{(\pi)}=J_{k+1, k}^{(\pi)}=(-1)^{k}$ and define

$$
\begin{aligned}
V_{k}(a, b)=V^{\prime}\left(a \mathcal{J}^{0}+b \mathcal{J}^{(\pi)}\right)_{k, k+1}= & \frac{a}{2 \pi\left(a^{2}-b^{2}\right)} \int_{\sigma} V^{\prime}(\lambda) \operatorname{sign} \lambda \frac{\left(\lambda^{2}-4 b^{2}\right)^{1 / 2}}{\left(4 a^{2}-\lambda^{2}\right)^{1 / 2}} d \lambda \\
& +\frac{(-1)^{k} b}{2 \pi\left(a^{2}-b^{2}\right)} \int_{\sigma} V^{\prime}(\lambda) \operatorname{sign} \lambda \frac{\left(4 a^{2}-\lambda^{2}\right)^{1 / 2}}{\left(\lambda^{2}-4 b^{2}\right)^{1 / 2}} d \lambda .
\end{aligned}
$$

It is easy to see, e.g., that

$$
\sum_{l_{1}, l_{2}} \mathcal{P}_{l_{1}, l_{2}}^{(2, k, 1)}(-1)^{l_{1}+l_{2}}=\left.\frac{1}{2} \frac{\partial^{2}}{\partial b^{2}} V_{k}(a, b)\right|_{a=1, b=0}=\frac{1}{2 \pi} \int_{-2}^{2} \frac{\lambda V^{\prime}(\lambda) d \lambda}{\sqrt{4-\lambda^{2}}}-\frac{1}{\pi} \int_{-2}^{2} \frac{V^{\prime}(\lambda) d \lambda}{\lambda \sqrt{4-\lambda^{2}}}=1 .
$$

Here we have used (2.11) for the first integral and (1.14) the second. Similarly

$$
\sum_{l_{1}, l_{2}} \mathcal{P}_{l_{1}, l_{2}}^{(2, k, 1)}(-1)^{l_{1}}=\left.\frac{1}{2} \frac{\partial^{2}}{\partial a \partial b} V_{k}(a, b)\right|_{a=1, b=0}=(-1)^{k}
$$

To compute the sum for $\mathcal{P}_{l_{1}, l_{2}, l_{3}}^{(3, k, 1)}$ let us observe that

$$
\sum_{l_{1}, l_{2}, l_{3}} \mathcal{P}_{l_{1}, l_{2}, l_{3}}^{(3, k, 1)}(-1)^{l_{1}+l_{2}+l_{3}}=\left.\frac{1}{6} \frac{\partial^{3}}{\partial b^{3}} V_{k}(1, b)\right|_{b=0}=\left.\frac{(-1)^{k}}{2} \frac{\partial^{2}}{\partial b^{2}} \frac{1}{\left(1-b^{2}\right)} I(b)\right|_{b=0},
$$

where

$$
\begin{aligned}
I(b)=\frac{1}{2 \pi} \int_{\sigma}\left(V^{\prime}(\lambda)-\lambda V^{\prime \prime}(0)\right) \operatorname{sign} \lambda \frac{\left(4-\lambda^{2}\right)^{1 / 2}}{\left(\lambda^{2}-4 b^{2}\right)^{1 / 2}} d \lambda & \\
& =\frac{1}{2 \pi} \int_{\sigma} V^{\prime}(\lambda) \operatorname{sign} \lambda \frac{\left(4-\lambda^{2}\right)^{1 / 2}}{\left(\lambda^{2}-4 b^{2}\right)^{1 / 2}} d \lambda-V^{\prime \prime}(0)\left(1-b^{2}\right) .
\end{aligned}
$$

Differentiating this expression, one can easily get the expression of (2.19).

To prove the last relation in (2.19) we use the symmetry arguments. Indeed, according to (3.23),

$$
\begin{gathered}
h\left(k, l_{1}\right)=\sum_{l_{2}}\left(\mathcal{P}_{l_{1}, l_{2}}^{(2, k, 1)}+\mathcal{P}_{l_{2}, l_{1}}^{(2, k, 1)}\right)(-1)^{l_{1}+l_{2}}=\sum_{j} v_{j}(i j d)^{2} \int_{s_{1}+s_{2}+s_{3}=1} d s_{1} d s_{2} d s_{3} \\
\left(\begin{array}{r}
u_{s_{1}}\left(k-l_{1}\right) f_{s_{2}, s_{3}}\left(k-l_{1}\right)+u_{s_{1}}\left(k-l_{1}-1\right) f_{s_{2}, s_{3}}\left(l_{1}-k_{1}-1\right) \\
\left.\quad+u_{s_{1}}\left(k-l_{1}\right) f_{s_{1}, s_{2}}\left(k-l_{1}\right)+u_{s_{1}}\left(k-l_{1}+1\right) f_{s_{1}, s_{2}}\left(l_{1}-k_{1}+1\right)\right),
\end{array}\right.
\end{gathered}
$$

where

$$
u_{s_{1}}(k-l)=\left(e^{i j d s_{1} \mathcal{J}^{(0)}}\right)_{k-l}, \quad f_{s_{2}, s_{3}}(l-k)=(-1)^{l}\left(e^{i j d s_{2} \mathcal{J}^{(0)}} \mathcal{J}^{(\pi)} e^{i j d s_{3} \mathcal{J}^{(0)}}\right)_{l, k} .
$$

Since both $u_{s_{1}}(k-l)$ and $f_{s_{2}, s_{3}}(l-k)$ are even functions with respect to $(l-k)$, after integration with respect to $s_{1}, s_{2}, s_{3}$ we get that

$$
h\left(k, l_{1}\right)=h\left(k-l_{1}\right)=h\left(l_{1}-k\right) \Rightarrow \sum_{l_{1}} h\left(k, l_{1}\right)\left(k-l_{1}\right)=0 .
$$


To prove (2.20) we define similarly to (3.27)

$$
\begin{aligned}
V_{k}^{(0)}(b)=v^{(0)}\left(\mathcal{J}^{(0)}+b \mathcal{J}^{(\pi)}\right)_{k, k} & =\frac{1}{\pi} \int_{\sigma} \frac{V^{\prime}(\lambda) \operatorname{sign} \lambda}{\left(4 b^{2}-\lambda\right)^{1 / 2} X(\lambda)} d \lambda \\
& =\frac{1}{\pi} \int_{\sigma} \frac{\left(V^{\prime}(\lambda)-\lambda V^{\prime \prime}(0)\right) \operatorname{sign} \lambda}{\left(4 b^{2}-\lambda\right)^{1 / 2} X(\lambda)} d \lambda+V^{\prime \prime}(0)
\end{aligned}
$$

Then

$$
\sum_{l_{1}, l_{2}} \mathcal{P}_{l_{1}, l_{2}}^{(2, k, 0)}(-1)^{l_{1}+l_{2}}=\left.\frac{1}{2} \frac{\partial^{2}}{\partial b^{2}} V_{k}^{0}(1, b)\right|_{b=0}=\frac{2}{\pi} \int_{-2}^{2} \frac{\left(V^{\prime}(\lambda)-\lambda V^{\prime \prime}(0)\right) d \lambda}{\lambda^{3} X(\lambda)}=2 P_{0}(0) .
$$

Proof of Lemma 3. Relation (2.30) can be written as

$$
d_{k}^{(2)}=2 x_{k}^{3}+\tilde{r}_{k}, \quad \tilde{r}_{k}=x_{k} k\left(2 P_{0}(0) n\right)^{-1}+r_{k}, \quad\left|\tilde{r}_{k}\right| \leq C_{*}\left(\tilde{m}_{k}|k| / n+\tilde{m}_{k}^{4}\right),
$$

where $C_{*}$ is independent of $N, n$ and we always can choose $C_{*}>1$. If $\tilde{m}_{k}<k^{-1}$ for all $k>n^{1 / 3}$, then (2.31) is fulfilled. If $\tilde{m}_{k}>k^{-1}$ for some $k>7 n^{1 / 3}$, we can apply Proposition 3 to $\left\{x_{j}\right\}_{|j| \leq M}$, with $M=k, M_{1}=\left[n^{1 / 3} / 2\right], \varepsilon^{3}=C_{*}\left(\tilde{m}_{k+2 M_{1}}\left(k+2 M_{1}\right) / n+m_{k+2 M_{1}}^{4}\right), \varepsilon_{1}=m_{k+2 M_{1}}$, because $M_{1}>2 / 3 \varepsilon^{-1}$. Then, since

$$
2 \varepsilon_{1} M_{1}^{-2}=8 \tilde{m}_{k+2 M_{1}} n^{-2 / 3}<C_{*} \tilde{m}_{k+2 M_{1}}\left(k+2 M_{1}\right) / n<\varepsilon^{3},
$$

we obtain by (2.34) that $8 \varepsilon^{3}=8 C_{*}\left(\tilde{m}_{k+2 M_{1}}\left(k+2 M_{1}\right) / n+m_{k+2 M_{1}}^{4}\right) \geq m_{k}^{3}$. Therefore at least one of the following inequalities holds

$$
8 C_{*} \tilde{m}_{k+2 M_{1}}\left(k+2 M_{1}\right) / n \geq m_{k}^{3} / 2 \quad \vee \quad 8 C_{*} m_{k+2 M_{1}}^{4} \geq m_{k}^{3} / 2
$$

Since according to Lemma $1\left|m_{k+2 M_{1}}\right| \leq C n^{-1 / 8} \log ^{1 / 4} n$ the second inequality yields

$$
\tilde{m}_{k+2 M_{1}}>2 \tilde{m}_{k}
$$

If the second inequality in (3.29) is false, then the first one holds. Write it as

$$
\tilde{m}_{k+2 M_{1}} \geq\left(16 C_{*}\right)^{-1} \tilde{m}_{k}\left[\tilde{m}_{k}^{2} n / k\right]\left[k /\left(k+n^{1 / 3}\right)\right] .
$$

Assume that for some $k>7 n^{1 / 3}$

$$
\tilde{m}_{k}^{2} n / k \geq 32 C_{*}
$$

Then (3.31)implies (3.30) and

$$
\tilde{m}_{k+2 M_{1}}^{2} n /\left(k+2 M_{1}\right) \geq 4\left(\tilde{m}_{k}^{2} n / k\right) \cdot\left(k /\left(k+2 M_{1}\right)\right)>32 C_{*} .
$$

Hence, we can repeat this procedure $l$ times with $l=[\log n]$. Then we obtain the inequality

$$
\tilde{m}_{k+[\log n] M_{1}}>2^{[\log n]} \tilde{m}_{k},
$$

which contradicts to Lemma 1, Thus, (3.32) is false and we have proved (2.31).

To prove (2.32) take any $k_{0}>n^{1 / 3}$ denote $\tilde{x}_{k}=x_{k-2 k_{0}}$ and, taking into account (2.30), apply (2.36) with $M=k_{0}$. Then since $f_{k}>\left(k_{0} / 2 P_{0}(0) n\right)$ we obtain (2.32).

Proof of Lemma 4 
Substituting in (3.5) $\phi(\lambda)=(z-\lambda)^{-1}$ we get easily the equation

$$
g_{n+k, n}^{2}(z)-\int \frac{V^{\prime}(\lambda)}{z-\lambda} \rho_{n+k, n}(\lambda) d \lambda=\delta_{n+k, n}(z)
$$

with $\delta_{n+k, n}(z)$ of the form (cf. (3.6) $)$

$$
\delta_{n+k, n}(z)=\frac{\left(J_{n+k}^{(n)}\right)^{2}}{n^{2}}\left(\left(R^{2}\right)_{n+k, n+k}(z)\left(R^{2}\right)_{n+k-1, n+k-1}(z)-\left(R^{2}\right)_{n+k, n+k-1}^{2}(z)\right),
$$

where $\left(R^{2}\right)_{k, j}=\left(z-\mathcal{J}^{(n)}\right)_{k, j}^{-2}$. Here we have used the Christoffel-Darboux formula in the numerator of the first integral in (2.43) . Let us transform

$$
\begin{gathered}
\int \frac{V^{\prime}(\lambda)}{z-\lambda} \rho_{n+k, n}(\lambda) d \lambda=g_{n+k, n}(z)\left(z V^{\prime \prime}(0)+z^{3} \frac{V^{(4)}(0)}{6}\right)-\int \frac{V^{\prime}(\lambda)}{\lambda} \rho_{n+k, n}(\lambda) d \lambda \\
-z^{2} \int \frac{V^{\prime}(\lambda)-\lambda V^{\prime \prime}(0)}{\lambda^{3}} \rho_{n+k, n}(\lambda) d \lambda-z^{4} \int \frac{V^{\prime}(\lambda)-\lambda V^{\prime \prime}(0)-\frac{1}{6} \lambda^{3} V^{(4)}(0)}{\lambda^{4}(z-\lambda)} \rho_{n+k, n}(\lambda) d \lambda \\
=g_{n+k, n}(z)\left(z V^{\prime \prime}(0)+z^{3} \frac{V^{(4)}(0)}{6}\right)-c_{k, n}^{(0)}-z^{2} c_{k, n}^{(2)}-z^{4} c_{k, n}^{(4)}(z) .
\end{gathered}
$$

Denote

$$
Q(\lambda)=\int \frac{V^{\prime}(\lambda)-V^{\prime}(\mu)}{\lambda-\mu} \rho(\mu) d \mu .
$$

Taking the limit $n \rightarrow \infty$ in (3.33) and using (1.13), we get for any $\lambda \in[-2,2]$

$$
\lambda^{4} P_{0}^{2}(\lambda)\left(\lambda^{2}-4\right)=\left[V^{\prime}(\lambda)\right]^{2}-4 Q(\lambda) \Rightarrow Q(\lambda)=\frac{1}{4}\left(\left[V^{\prime}(\lambda)\right]^{2}+\lambda^{4} P_{0}^{2}(\lambda)\left(4-\lambda^{2}\right)\right) .
$$

Therefore, denoting $v^{(0)}(\lambda)=V^{\prime}(\lambda) \lambda^{-1}$, we get

$$
\begin{aligned}
& c_{k, n}^{(0)}=Q(0)+\tilde{c}_{n}^{(0)}+c_{k}=\tilde{c}_{n}^{(0)}+c_{k}, \\
& \tilde{c}_{n}^{(0)}=\int v^{(0)}(\lambda)\left(\rho_{n, n}(\lambda)-\rho(\lambda)\right) d \lambda, \quad c_{k}= \pm n^{-1} \sum_{j=1}^{|k|} v^{(0)}\left(\mathcal{J}^{(n)}\right)_{n \pm j, n \pm j} .
\end{aligned}
$$

Here and below in the proof of Lemma 4 the sign \pm corresponds to the sign of $k$. Repeating the argument of Lemma 2 for the function $v^{(0)}(\lambda)$, we obtain

$$
v^{(0)}\left(\mathcal{J}^{(n)}\right)_{n \pm j, n \pm j}=x_{j}^{2} \sum_{\left|l_{1}\right|,\left|l_{2}\right|<|k|+n^{1 / 3}} \mathcal{P}_{l_{1}, l_{2}}^{(2, k, 0)}(-1)^{l_{1}+l_{2}}+y_{j} \sum_{\left|l_{1}\right|<|k|+n^{1 / 3}} \mathcal{P}_{l_{1}}^{(0)}+n^{1 / 6} O\left((k / n)^{3 / 2}\right) .
$$

Hence, using (2.20), we get (2.42). Now let us observe that

$$
\int \frac{V^{\prime}(\lambda)-\lambda V^{\prime \prime}(0)}{\lambda^{3}} \rho(\lambda) d \lambda=\left.\frac{1}{2} \frac{d^{2}}{d \mu^{2}} Q(\mu)\right|_{\mu=0}=\frac{1}{4}\left(V^{\prime \prime}(0)\right)^{2} .
$$

Hence,

$$
c_{k, n}^{(2)}=\frac{1}{4}\left(V^{\prime \prime}(0)\right)^{2}+\tilde{c}_{n}^{(2)} \pm \sum_{j=1}^{|k|} v^{(2)}\left(\mathcal{J}^{(n)}\right)_{n \pm j, n \pm j}, \quad\left(v^{(2)}(\lambda)=\left(V^{\prime}(\lambda)-\lambda V^{\prime \prime}(0)\right) \lambda^{-3}\right),
$$


where

$$
\tilde{c}_{n}^{(2)}=\int v^{(2)}(\lambda)\left(\rho_{n, n}(\lambda)-\rho(\lambda)\right) d \lambda
$$

Using Corollary 2 from Lemma 3 , we get

$$
v^{(2)}\left(\mathcal{J}^{(n)}\right)_{n+k, n+k}=\int \frac{v^{(2)}(\lambda) d \lambda}{\sqrt{4-\lambda^{2}}}+O(|k| / n)+O\left(n^{-2 / 3}\right)=P_{0}(0)+O(|k| / n)+O\left(n^{-2 / 3}\right) .
$$

Therefore

$$
c_{k, n}^{(2)}=\frac{1}{4}\left(V^{\prime \prime}(0)\right)^{2}+P_{0}(0) \frac{k}{n}+O\left(k^{2} / n^{2}\right)+\tilde{c}_{n}^{(2)} .
$$

Now we apply (3.10) to

$$
v^{(4)}(\lambda, z)=\frac{V^{\prime}(\lambda)-\lambda V^{\prime \prime}(0)-\frac{1}{6} \lambda^{3} V^{(4)}(0)}{\lambda^{4}(z-\lambda)}
$$

We get

$$
\begin{gathered}
c_{k, n}^{(4)}(z)=\int v^{(4)}(\lambda, z) \rho(\lambda) d \lambda \pm n^{-1} \sum_{j=1}^{|k|} v^{(4)}\left(\mathcal{J}^{(n)}\right)_{n \pm j, n \pm j}+O\left(n^{-1 / 2} \log ^{1 / 2} n /|\Im z|\right) \\
=\frac{Q^{(4)}(0)}{4 !}+O(z)+O\left(n^{-1 / 2} \log ^{1 / 2} n /|\Im z|\right)+O(k / n) \\
=\frac{1}{4 !}\left(24 P_{0}^{2}(0)+2 V^{\prime}(0) V^{\prime \prime}(0)\right)+O(z)+O\left(n^{-1 / 2} \log ^{1 / 2} n /|\Im z|\right)+O(k / n),
\end{gathered}
$$

where the last equality follows from (3.36). Now, substituting (3.37)-(3.39) in (3.35), we find

$$
g_{n+k, n}(z)=\frac{1}{2}\left(z V^{\prime \prime}(0)+z^{3} \frac{V^{(4)}(0)}{6}\right)-\left(-P_{0}^{2}(0) z^{4}-\frac{k}{n} P_{0}(0) z^{2}-c_{k}+\delta_{n+k, n}(z)-\tilde{\delta}_{n+k, n}(z)\right)^{1 / 2}
$$

with $c_{k}$ defined by (2.42),$\delta_{n+k, n}(z)$ defined by (3.34) and

$$
\tilde{\delta}_{n+k, n}(z)=\tilde{c}_{n}^{(0)}+z^{2} \tilde{c}_{n}^{(2)}+z^{2}\left(O\left(n^{-4 / 3}\right)+O\left(k^{2} / n^{2}\right)\right)+O\left(z^{5}\right)+\frac{z^{4}}{|\Im z|} O\left(n^{-1 / 2} \log ^{1 / 2} n\right) .
$$

Since (2.41) follows from (3.40), we are left to estimate $\tilde{c}_{n}^{(0)}, \tilde{c}_{n}^{(2)}$ and $\delta_{n+k, n}(z)$.

Taking into account (3.34), to estimate $\delta_{n+k, n}(z)$ we need to estimate $\left(R^{2}\right)_{n+k, n+k}$ and $\left(R^{2}\right)_{n+k, n+k-1}$. Let us take $N^{\prime}=k+\log ^{2} n n^{1 / 3}$, and consider $\tilde{\mathcal{J}}\left(N^{\prime}\right)$ defined by (2.3) and

$$
R^{(1)}(z)=\left(z-\tilde{\mathcal{J}}^{(0)}-\tilde{\mathcal{J}}\left(N^{\prime}\right)\right)^{-1} .
$$

Then, using the resolvent identity (2.64) and (2.62), we get for any $z: \Im z>n^{-1 / 3}$

$$
\left|\left(R^{2}\right)_{n+k, n+k}-\left(R^{(1)} * R^{(1)}\right)_{k, k}(z)\right| \leq C e^{-C \log ^{2} n} .
$$

Applying the resolvent identity (2.64) to $R^{(0)}=\left(z-\mathcal{J}^{(0)}\right)^{-1}$ and $R^{(1)}(z)$ defined above, we get

$$
\begin{aligned}
& \left|\left(R^{(1)} * R^{(1)}\right)_{k, k}(z)-\left(R^{(0)} * R^{(0)}\right)_{k, k}(z)\right| \\
& \quad \leq 2\left(\left(R^{(0)}(z) * R^{(0)}(\bar{z})\right)_{k, k}+|\Im z|^{-2}\left(R^{(0)}(z) * \tilde{J}^{2}\left(N^{\prime}\right) * R^{(0)}(\bar{z})\right)_{k, k}\right) \\
& \quad \leq \frac{C}{|\Im z|}+\frac{C|k|}{n|\Im z|^{3}}+\frac{2}{n|\Im z|^{2}} \sum\left|R^{(0)}(z)_{k, j}\right|^{2}|k-j| \leq \frac{C}{|\Im z|}\left(1+\frac{|k|}{n|\Im z|^{2}}+\frac{1}{n|\Im z|^{3}}\right) .
\end{aligned}
$$


Here we have used that for $|\Im z| \leq 1$

$$
\left(R^{(0)}(z) * R^{(0)}(\bar{z})\right)_{k, k}=\sum\left|R_{k, j}^{(0)}(z)\right|^{2}=\frac{1}{\pi} \int_{-2}^{2} \frac{d \lambda}{|z-\lambda|^{2} \sqrt{4-\lambda^{2}}} \leq \frac{C}{|\Im z|} .
$$

Substituting (3.43) in (3.34), we get the first estimate in (2.43).

To estimate $\tilde{c}_{n}^{(0)}$ and $\tilde{c}_{n}^{(2)}$ we subtract from (3.33) the same equation for $k:=k-1$ and multiply the result by $n$ (see the proof of Lemma 1 for the details). Then we get

$$
2 g_{n+k, n}(z) R_{n+k, n+k}(z)=\int \frac{V^{\prime}(\lambda)}{z-\lambda}\left(\psi_{n+k}^{(n)}(\lambda)\right)^{2} d \lambda-\delta_{n+k, n}^{(R)}(z),
$$

where

$$
\delta_{n+k, n}^{(R)}(z)=\frac{1}{n} \sum_{j=1}^{\infty} R_{n+k, j}(z) R_{j, n+k}(z)-\frac{2}{n} \sum_{j=1}^{n+k} R_{n+k, j}(z) R_{j, n+k}(z) .
$$

Using the same trick as in (3.42), we get

$$
\delta_{k, n}^{(R)}(z)=\frac{1}{n} \sum_{j=1}^{\infty} R_{k, j}^{(1)}(z) R_{j, k}^{(1)}(z)-\frac{2}{n} \sum_{j=1}^{k} R_{k, j}^{(1)}(z) R_{j, k}^{(1)}(z)+O\left(e^{-C \log ^{2} n}\right) .
$$

Besides, since $R_{k, j}^{(0)}(z)$ is an even function of $(j-k)$, we observe that

$$
0=\frac{1}{n} \sum_{j=-\infty}^{\infty} R_{k, j}^{(0)}(z) R_{j, k}^{(0)}(z)-\frac{2}{n} \sum_{j=-\infty}^{k} R_{k, j}^{(0)}(z) R_{j, k}^{(0)}(z)+\frac{1}{n}\left(R_{k, k}^{(0)}(z)\right)^{2}
$$

Hence, to estimate $\delta_{n+k, n}^{(R)}(z)$ it is enough to estimate the difference between r.h.s. of the last two formulas. Using (3.43) for the difference of the first sums, similar bound for the difference of the second sums, and the bound $\left|R_{k, k}^{(0)}(z)\right|=\left|z^{2}-4\right|^{-1 / 2} \leq C$ for $|z|<1$, we get

$$
\left|\delta_{n+k, n}^{(R)}(z)\right| \leq \frac{C}{n|\Im z|}\left(1+\frac{|k|}{n|\Im z|^{2}}+\frac{1}{n|\Im z|^{3}}\right) .
$$

Now performing transformations (3.35) for the integral in the r.h.s. of (3.44), we can rewrite it as

$$
R_{n+k, n+k}(z)\left(2 g_{n+k, n}(z)-V^{\prime \prime}(0) z-V^{(4)}(0) z^{3} / 6\right)=a_{k, n}^{(2)} z^{2}-a_{k, n}^{(0)}+\delta_{n+k, n}^{(R)}(z)+O\left(z^{4}\right),
$$

where

$$
\begin{aligned}
& a_{k, n}^{(0)}=v^{(0)}\left(\mathcal{J}^{(n)}\right)_{n+k, n+k}=2 n^{-2 / 3} P_{0}(0) q_{n}^{2}\left(\frac{k}{n^{1 / 3}}\right)+\frac{k}{2 n}+O\left(n^{-1}\right) \\
& a_{k, n}^{(2)}=v^{(2)}\left(\mathcal{J}^{(n)}\right)_{n+k, n+k}=P_{0}(0)+O\left(n^{-2 / 3}\right) .
\end{aligned}
$$

Let us take $k>0$ and change the variable $z=\tilde{\varepsilon} \zeta$ with $\tilde{\varepsilon}^{2}=k / P_{0}(0) n$ in (3.46). Then, using (3.40), we obtain from (3.46)

$$
R_{n+k, n+k}(\tilde{\varepsilon} \zeta)=\frac{\zeta^{2}+\frac{1}{2}+2 P_{0}(0) \frac{n^{1 / 3}}{k} q_{n}^{2}\left(\frac{k}{n^{1 / 3}}\right)+\tilde{\varepsilon}^{-2} P_{0}^{-1}(0) \delta_{n+k, n}^{(R)}(\tilde{\varepsilon} \zeta)+o(1)}{2 i\left(\zeta^{4}+\zeta^{2}+\tilde{\varepsilon}^{-4} P_{0}^{-2}(0)\left(c_{k}+\tilde{\delta}_{n+k, n}(\tilde{\varepsilon} \zeta)-\delta_{n+k, n}(\tilde{\varepsilon} \zeta)\right)\right)^{1 / 2}}:=\frac{R_{1}(\zeta)}{R_{2}^{1 / 2}(\zeta)} .
$$


In view of (3.45)

$$
\tilde{\varepsilon}^{-2}\left|\delta_{k, n}^{(R)}(\tilde{\varepsilon} \zeta)\right| \rightarrow 0, \quad \text { as } \quad \frac{k}{n^{1 / 3}} \rightarrow \infty, \quad \Im \zeta>d
$$

with any fixed $d$ (see (3.45)). Besides, $q_{n}^{2}(x) \rightarrow 0$, as $x \rightarrow \infty$, because of (2.32). Therefore there exists some fixed $l_{0}>0$, such that for $k>l_{0} n^{1 / 3}$ and any $\zeta: \Im \zeta>1 / 4$

$$
\left|2 P_{0}(0) \frac{n^{1 / 3}}{k} q_{n}^{2}\left(\frac{k}{n^{1 / 3}}\right)+\tilde{\varepsilon}^{-2} P_{1}^{-1}(0) \delta_{n+k, n}^{(R)}(\tilde{\varepsilon} \zeta)\right|<\frac{1}{4}<\min _{|\zeta-i / \sqrt{2}|=1 / 4}\left|\zeta^{2}+\frac{1}{2}\right| .
$$

Then according to the Rouchet theorem $R_{1}(\zeta)$ has a root inside the circle $\mathcal{B}$ of radius $1 / 4$ centered at $i / \sqrt{2}$. Thus, if $R_{2}(\zeta)$ has no roots of the second order inside $\mathcal{B}$, then similarly to the proof of Lemma 1 we obtain a contradiction with (3.17). Therefore, using the first inequality of (2.43), (3.41) and (3.45) we conclude that there exists an absolute constant $C_{0}$, such that

$$
\left|\tilde{c}_{n}^{(0)}\right|,\left|\tilde{c}_{n}^{(2)}\right|^{2} \leq C_{0} n^{-4 / 3} .
$$

These bounds and (3.41) prove the second estimate of (2.43).

\section{Appendix}

Proof of Proposition 1. Using the spectral theorem and Proposition 7, we get

$$
\left|v\left(\mathcal{J}^{(n)}\right)_{n+k, n+k+\delta}-\tilde{v}\left(\mathcal{J}^{(n)}\right)_{n+k, n+k+\delta}\right|=\left|\int(v(\lambda)-\tilde{v}(\lambda)) \psi_{n+k}^{(n)}(\lambda) \psi_{n+k+\delta}^{(n)}(\lambda) d \lambda\right| \leq C e^{-n C \varepsilon} .
$$

Let us represent $\tilde{v}(\lambda)$ by its Fourier expansion

$$
\tilde{v}(\lambda)=\sum_{j=-\infty}^{\infty} v_{j} e^{i j d \lambda}, \quad d=\frac{\pi}{2+\varepsilon} .
$$

Then we have

$$
\tilde{v}\left(\mathcal{J}^{(n)}\right)=\sum_{j=-\infty}^{\infty} v_{j} e^{i j d \mathcal{J}^{(n)}}=\sum_{|j| \leq c M} v_{j} e^{i j d \mathcal{J}^{(n)}}+O\left(M^{-\ell+1 / 2}\right),
$$

where $c$ is some absolute constant which we will choose later. The bound for the remainder term in the last formula follows from the estimate

$\left\|\sum_{|j|>c M} v_{j} e^{i j d \mathcal{J}^{(n)}}\right\| \leq \sum_{|j|>c M}\left|v_{j}\right| \leq\left(\sum_{|j|>c M}\left|v_{j}\right|^{2}|j|^{2 \ell}\right)^{1 / 2}\left(\sum_{|j|>c M}|j|^{-2 \ell}\right)^{1 / 2} \leq\left\|v^{(\ell)}\right\|_{2}(c M)^{-\ell+1 / 2}$.

Consider now $N^{\prime}=[N+M]+1$ and denote by $\mathcal{J}^{\left(n, N^{\prime}\right)}$ the matrix whose entries coincide with that of $\mathcal{J}^{(n)}$ with the only exception $\mathcal{J}_{n \pm N^{\prime}, n \pm N^{\prime}+1}^{\left(n, N^{\prime}\right)}=0$. We will use the identity, valid for any matrices $\mathcal{J}_{1}, \mathcal{J}_{2}$

$$
e^{i t \mathcal{J}_{2}}-e^{i t \mathcal{J}_{1}}=\int_{0}^{t} e^{i(t-s) \mathcal{J}_{1}}\left(\mathcal{J}_{2}-\mathcal{J}_{1}\right) e^{i s \mathcal{J}_{2}} d s
$$


Let us take $|k|<N$ and apply (4.3) to $\mathcal{J}_{n+k, n+k+\delta}^{(n)}$ and $\mathcal{J}_{n+k, n+k+\delta}^{\left(n, N^{\prime}\right)}$. Then we get

$$
\begin{aligned}
& v\left(\mathcal{J}^{(n)}\right)_{n+k, n+k+\delta}-v\left(\mathcal{J}^{(n, N)}\right)_{n+k, n+k+\delta} \\
& =\int_{0}^{t} d s \sum_{|j| \leq M} v_{j} \sum_{ \pm}\left(\left(e^{i j d(t-s) \mathcal{J}^{\left(n, N^{\prime}\right)}}\right)_{n+k, n \pm N^{\prime}} \mathcal{J}_{n \pm N^{\prime}, n \pm N^{\prime}+1}^{(n)}\left(e^{i s \mathcal{J}^{(n)}}\right)_{n \pm N^{\prime}+1, n+k+\delta}\right. \\
& \left.\quad+\left(e^{i j d(t-s) \mathcal{J}^{\left(n, N^{\prime}\right)}}\right)_{n+k, n \pm N^{\prime}+1} \mathcal{J}_{n \pm N^{\prime}+1, n \pm N^{\prime}}^{(n)}\left(e^{i s \mathcal{J}^{(n)}}\right)_{n \pm N^{\prime}, n+k+\delta}\right)+O\left(M^{-\ell+1 / 2}\right)
\end{aligned}
$$

Now we use the bound, valid for any Jacobi matrix $\mathcal{J}$ with coefficients $J_{k, k+1}=J_{k+1, k}=a_{k} \in \mathbf{R}$, $\left|a_{k}\right| \leq A$. Then there exist positive constants $C_{0}, C_{1}, C_{2}$, depending on $A$ such that the matrix elements of $e^{i t \mathcal{J}}$ satisfy the inequalities:

$$
\left|\left(e^{i t \mathcal{J}}\right)_{k, j}\right| \leq C_{0} e^{-C_{1}|k-j|+C_{2} t} .
$$

This bound follows from the representation

$$
\left(e^{i t \mathcal{J}}\right)_{k, j}=\frac{1}{2 \pi i} \oint_{l} e^{i t z} \tilde{R}_{k, j}(z) d z
$$

where $\tilde{R}=(z I-\mathcal{J})^{-1}$, and from (2.62).

Using (4.5) in (4.4), we get for any $c<C_{0}\left(C_{1} d\right)^{-1}$

$$
v\left(\mathcal{J}^{(n)}\right)_{n+k, n+k+\delta}-v\left(\mathcal{J}^{\left(n, N^{\prime}\right)}\right)_{n+k, n+k+\delta}=O\left((c M)^{-\ell+1 / 2}\right) .
$$

Similarly (see definitions (2.2) and (2.3) ):

$$
v\left(\mathcal{J}^{(0)}+\tilde{\mathcal{J}}\right)_{k, k+\delta}-v\left(\mathcal{J}^{\left(n, N^{\prime}\right)}\right)_{n+k, n+k+\delta}=O\left((c M)^{-\ell+1 / 2}\right) .
$$

These two bounds give us (2.4).

Proof of Proposition 3. Assume that $\left|\tilde{x}_{k}\right|>\varepsilon$ for some $k$. Without loss of generality we can assume that $\tilde{x}_{k}>0$. Then due to (2.33)

$$
\tilde{x}_{k+1}-2 \tilde{x}_{k}+\tilde{x}_{k-1}>\tilde{x}_{k}^{3}
$$

Consider first the case when also $\tilde{d}_{k}^{(1)}=\tilde{x}_{k+1}-\tilde{x}_{k}>0$. Then by induction for any $M-k>i>0$ we have $\tilde{d}_{k+i}^{(1)}>\tilde{d}_{k}^{(1)}, \tilde{x}_{k+i}>\tilde{x}_{k}$ and $\tilde{d}_{k+i}^{(2)}>\tilde{x}_{k}^{3}$. Hence

$$
\varepsilon_{1}>\tilde{x}_{k+M_{1}}>\tilde{x}_{k}+\tilde{x}_{k}^{3} M_{1}^{2} / 2 .
$$

If $\tilde{d}_{k}^{(1)}<0$, then according to (2.33) we have $\tilde{x}_{-k-1}>\tilde{x}_{k}$ and we obtain (2.34) moving from $k$ in the negative direction.

Similarly, assume that at some point $|k| \leq M-2 M_{1}$

$$
\tilde{d}_{k}^{(1)}>4 \max \left\{\varepsilon^{2},\left(2 \varepsilon_{1} M_{1}^{-2}\right)^{2 / 3}\right\}=: 4 \mu^{2} .
$$

Since $\left|\tilde{d}_{k}^{(2)}\right| \leq 3 \mu^{3}$ because of (2.33) and the first inequality of (2.34), we have for any $|i|<i_{0}:=$ $[2 /(3 \mu)] \leq[2 /(3 \varepsilon)] \leq M_{1}$

$$
\tilde{d}_{k+i}^{(1)}>\tilde{d}_{k}^{(1)} / 2 \Rightarrow \mu \geq\left|\tilde{x}_{k+s i_{0}}\right|>i_{0} \tilde{d}_{k}^{(1)} / 2=3 \mu^{-1} d_{k}^{(1)},
$$


where $s=\operatorname{sign} \tilde{x}_{k}$. The last inequality here contradicts to (4.6). Hence, (4.6) is false and we obtain the second inequality of (2.34).

To prove (2.36) observe that if we consider two $(2 M+1) \times(2 M+1)$ Jacobi matrices $J^{(f)}$ and $J^{(d)}$ with entries

$$
J^{(f)}=D^{(f)}-J^{(0)}, \quad J^{(d)}=D^{(d)}-J^{(0)} D_{k, j}^{(f)}=\delta_{k, j}\left(2+f_{k}\right), \quad D_{k, j}^{(d)}=\delta_{k, j}\left(2+d^{2}\right), \quad|k| \leq 2 M
$$

then, using the Neumann expansion for their inverse we get

$$
0 \leq\left(J^{(f)}\right)_{k, j}^{-1} \leq\left(J^{(f)}\right)_{k, j}^{-1} \leq(2 d)^{-1} e^{-d|k-j|} .
$$

Hence, rewriting (2.35) as

$$
\left(J^{(f)} \tilde{x}\right)_{k}=-\tilde{r}_{k}+\delta_{k, 2 M} \tilde{x}_{2 M+1}+\delta_{k,-2 M} \tilde{x}_{-2 M-1}
$$

we get (2.36) from (4.7).

Proof of Proposition 4. It is evident that it is enough to prove (2.52) for the case when $2 P_{0}(0)=1$ in (1.17) and (2.52). Hence, below we consider this case. For $x>0$ the statement is evident. Let $f(x)=\sqrt{-x / 6}-q(x)$ and $x_{0}$ be the first negative root of $f$. Since it is known (see [12] ) that $q(x)=A i(x)(1+o(1))$ as $x \rightarrow+\infty$, we conclude that $q(0)>A i(0)=0.355028 \ldots>$ $3^{2 / 3} / 6$ (for $A i(0)$ see [1]) Besides, it is known that $q(x)>0, q^{\prime}(x)<0$ (see [12]) and so

$$
\sqrt{-x_{0} / 6}=q\left(x_{0}\right)>q(0)>3^{2 / 3} / 6 \Rightarrow x_{0}<-3^{1 / 3} / 2 .
$$

But for any point $x \leq x_{0}<-3^{1 / 3} / 2$ in which $q(x) \geq \sqrt{-x / 6}$

$$
q^{\prime \prime}(x) \leq \sqrt{-x / 6}(x-x / 3) \leq-\frac{2}{3} \sqrt{-x_{0}^{3} / 6}<-\left(4 \sqrt{6\left(-x_{0}\right)^{3}}\right)^{-1} \leq(\sqrt{-x / 6})^{\prime \prime} .
$$

Therefore $f^{\prime \prime}(x)>0$ for $x \geq x_{0}$. Since by definition $f\left(x_{0}\right)=0, f^{\prime}\left(x_{0}\right) \leq 0$ (because $f(0)<0$ an $x_{0}$ is the first root of $f$ ) we conclude that $f(x)<0$ for any $x<x_{0}$ that contradicts to (1.18). Thus we have proved that the left hand side of (2.52) is always positive. But since it tends to infinity as $x \rightarrow \pm \infty$, we conclude that there exists positive $\delta$, satisfying (2.52).

Proof of Proposition [6. We use the estimate for matrix elements of the resolvent of an arbitrary Jacobi matrix $\mathcal{J}$, with entries $\left|\mathcal{J}_{j, j+1}\right| \leq A$ :

$$
\left|\mathcal{R}_{k, j}(z)\right| \leq \frac{C_{1}^{\prime}}{|\Im z|} e^{-C_{2}^{\prime}|\Im z||k-j|},
$$

This estimate is similar to well-known Combes- Thomas estimates for Schrödinger operator (see e.g. [19]).

Let $\mathcal{J}^{(k, M)}$ be the Jacobi matrix, whose entries coincide with that for $\mathcal{J}$ with the only exceptions $\mathcal{J}_{k \pm M, k \pm M+1}^{(k, M)}=\mathcal{J}_{k \pm M+1, k \pm M}^{(k, M)}=0$, and $\mathcal{R}^{(k, M)}=\left(z-\mathcal{J}^{(k, M)}\right)^{-1}$. Then, by the resolvent identity (2.64)

$$
\mathcal{R}_{k, j}-\mathcal{R}_{k, j}^{(k, M)}=\sum_{ \pm}\left(\mathcal{R}_{k, k \pm M}^{(k, M)} \mathcal{J}_{k \pm M, k \pm M+1} \mathcal{R}_{k \pm M+1, j}+\mathcal{R}_{k, k \pm M+1}^{(k, M)} \mathcal{J}_{k \pm M+1, k \pm M} \mathcal{R}_{k \pm M, j}\right)
$$

Since $\mathcal{J}^{(k, M)}$ has a block structure, its resolvent $\mathcal{R}^{(k, M)}$ also has a block structure and its coefficients $\mathcal{R}_{k, j}^{(k, M)}$ do not depend on $\mathcal{J}_{j, j+1}$ with $|j-k|>M$. Hence, we can apply (4.8) to $\mathcal{R}_{k, j}^{(k, M)}, \mathcal{R}_{k, k \pm M}^{(k, M)}$ and $\mathcal{R}_{k, k \pm M+1}^{(k, M)}$. Then we get (2.62). Proposition 6 is proved.

Acknowledgements. The author is grateful to Prof. L.Pastur and Prof. A.Kuijalaars for the fruitful discussion. The author acknowledges also the INTAS Research Network 03-51-6637 for the financial support. 


\section{References}

[1] Abramowitz M., Stegun, I. Handbook of Mathematical Functions, Dover, N.Y., 1972

[2] S.Albeverio, L.Pastur and M.Shcherbina. On Asymptotic Properties of the Jacobi Matrix Coefficients. Matem. Fizika, Analiz, Geometriya 4, 263-277 (1997)

[3] Albeverio, S., Pastur, L., Shcherbina, M.: On the $1 / n$ expansion for some unitary invariant ensembles of random matrices. Commun. Math. Phys. 224, 271-305 (2001).

[4] Bleher, P., Its, A.: Double Scaling Limit in the Random Matrix Model: the Riemann-Hilbert Approach. Commun.Pure Appl.Math 56, 433-516 (2003)

[5] Boutet de Monvel, A., Pastur L., Shcherbina M.: On the statistical mechanics approach in the random matrix theory. Integrated density of states. J. Stat. Phys. 79, 585-611 (1995)

[6] M. Bowick, E. Brezin, Universal scaling of the tail of the density of eigenvalues in random matrix models, Phys. Lett. B 268, 21-28 (1991).

[7] Claeys, T., Kuijalaars, A.B.J.: Universality of the double scaling limit in random matrix models, preprint arXiv:math-ph/0501074

[8] Deift, P., Kriecherbauer, T., McLaughlin, K., Venakides, S., Zhou, X.: Uniform asymptotics for polynomials orthogonal with respect to varying exponential weights and applications to universality questions in random matrix theory. Commun. Pure Appl. Math. 52 ,1335-1425 (1999)

[9] Deift, P., Kriecherbauer, T., McLaughlin, K., Venakides, S., Zhou, X.: Strong asymptotics of orthogonal polynomials with respect to exponential weights. Commun. Pure Appl. Math. 52 1491-1552 (1999)

[10] Dyson, D.J.: A Class of Matrix Ensembles. J.Math.Phys.,13 90-107. 1972

[11] P. Forrester, The spectrum edges of random matrix ensembles, Nucl. Phys. B 402 (1993) 709-728.

[12] Hastings, S.P., McLeod, J.B.: A boundary value problem assiciated with the second Painleve transcendent and the Korteweg de Vries equation.Arch. Rational Mech.Anal, 73, 31-52 (1980)

[13] Johansson, K., On fluctuations of eigenvalues of random Hermitian matrices. Duke Math. J. 91, 151-204 (1998)

[14] Levitan,B.M., Sargsyan, I.S. Introduction to Spectral Theory, Translation Math. Monographs,39, AMS, Providence, RI, 1976

[15] M.L.Mehta, M.L.: Random Matrices. New York: Academic Press, 1991

[16] Muskhelishvili N.I. Singular Integral Equations. P.Noordhoff.- Groningen 1953.

[17] Pastur, L., Shcherbina, M.: Universality of the local eigenvalue statistics for a class of unitary invariant random matrix ensembles. J. Stat. Phys. 86, 109-147 (1997)

[18] Pastur, L., Shcherbina, M.:On the edge universality of the local eigenvalue statistics of matrix models. Matematicheskaya fizika, analiz, geometriya 10, N3, 335-365 (2003)

[19] Reed,M., Simon,B.:Methods of Modern Mathematical Physics, Vol.IV, Academic Press: New York, 1978

[20] C.A. Tracy, H. Widom, Level spacing distributions and the Airy kernel, Comm. Math. Phys. 159, 151-174 (1994) 